\title{
Differenzialdiagnose „Schwindel“
}

\section{Differential Diagnosis "Vertigo and Dizziness"}

\author{
S. K. Plontke , L. E. Walther
}

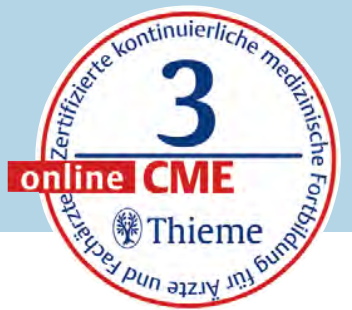

\section{Zusammenfassung \\ $\nabla$}

„Schwindel“ ist ein Symptom mit interdisziplinärer Bedeutung. Die Differenzierung und Einordnung von Schwindelsyndromen erfordert jedoch auch fachübergreifende Kenntnisse. Gerade beim subjektiven Symptom „Schwindel“ ist eine subtile Analyse der zugrunde liegenden Beschwerden erforderlich. Internationale Krankheitsdefinitionen sind bei der Differenzialdiagnose von Schwindelsyndromen heute ein unverzichtbares Hilfsmittel. Mit einfachen Mitteln können heute Augenbewegungsstörungen und Nystagmus untersucht und fachspezifisch zugeordnet werden. Screeninguntersuchungen (z.B. Kopfimpulstest) sind heute ein obligates Instrument bei der Untersuchung von Patienten mit Schwindelsyndromen als Notfall. Mit objektiven diagnostischen Verfahren (kalorische Nystagmografie, Videokopfimpulstest, vestibulär evozierte myogene Potenziale) kann das Ausmaß einer funktionelle Beeinträchtigung der fünf vestibulären Rezeptoren heute seiten- und rezeptorspezifisch aber auch unter dynamischen Aspekten beurteilt werden.

\section{Abstract \\ $\nabla$}

Vertigo and dizziness are symptoms of interdisciplinary dimension. However, the differentiation and classification of vertigo syndromes also require experience and multidisciplinary knowledge. Since the clinical syndrome is subjective, a detailed analysis of the complaints underlying is required. International disease definitions are an indispensable tool in the differential diagnosis of vertigo syndromes today. With simple diagnostic tools eye movement disorders and nystagmus can be examined and assigned to specific vestibular disorders today. Screening tests (e.g. head impulse test) are now an important instrument in the investigation of patients with vertigo syndromes in case of emergency. With objective diagnostic methods (caloric irrigation, video head impulse test, vestibular evoked myogenic potentials) the degree of functional impairment of the five vestibular receptors can be assessed quantitatively. Furthermore, in vestibulopathies, a receptor and side-specific diagnostic assessment can be performed even with regard to dynamic aspects.

\section{Facharztwissen HNO}

Dieser Beitrag aus der Serie „Facharztwissen HNO“ in der Laryngo-Rhino-Otologie widmet sich dem Thema Differenzialdiagnose „Schwindel“. Über das Jahr verteilt erscheinen in unserer Rubrik „Facharztwissen HNO“ alle 2 Monate didaktisch aufbereitete Übersichtsartikel. Diese greifen die Hauptthemen der Kursreihe „Facharztwissen HNO“ auf, die im Wechsel an den HNO-Kliniken in Dresden, Erfurt, Jena, Leipzig, Magdeburg und Halle stattfindet (jährliche Veranstaltung der Professoren Andreas Dietz, Orlando Guntinas-Lichius, Thomas Zahnert, Dirk Eßer, Christoph Arens und Stefan Plontke). Die- se Kursreihe zielt erstrangig auf Assistenten in der Weiterbildung HNO-Heilkunde ab und soll in möglichst persönlichem Rahmen eine direkte Diskussion der Lehrinhalte im Rahmen eines interaktiven Workshops ermöglichen. Die Autoren haben sich zur Aufgabe gestellt, die Inhalte praxisgerecht mit Rücksicht auf aktuelle, wissenschaftlich belastbare Entwicklungen anzubieten. Der Inhalt des HNO-Facharztwissens unterliegt einem ständigen Fluss, sodass durch das Konsensusverfahren der 6 genannten ausgewiesenen Fachvertreter die Aktualität und Belastbarkeit des angebotenen Wissens angestrebt wird.

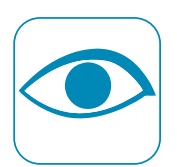

\section{Video online!}

Sie finden die Videos unter

www.thieme-connect.de/ products bei Ihrer LaryngoRhino-Otologie 


\section{Einleitung}

„Schwindel“ ist ein häufiges, fachübergreifendes Symptom in der klinischen Praxis. Die Vielzahl und die Komplexität der Interaktion der am Körpergleichgewicht beteiligten Bestandteile ist die Ursache für die Häufigkeit von Schwindelsyndromen. Daher ist für die Abklärung von „Schwindel“ immer eine interdisziplinäre Sichtweise erforderlich.

Eine Differenzierung und Klassifikation peripherer und zentraler vestibulärer sowie nichtvestibulärer Ursachen ist aus methodischer und didaktischer Sicht sinnvoll. Insbesondere in der Primärdiagnostik des Symptoms „Schwindel“ ist in der klinischen Praxis eine Gesamtschau auf Gleichgewichtsstörungen bzw. vestibuläre Syndrome mit differenzialdiagnostischen und fachübergreifenden Aspekten erforderlich.

Daher muss der HNO-Arzt in der Lage sein, differenzialdiagnostisch Zusammenhänge zu erkennen, welche durch Beeinträchtigungen der vestibulären Bereiche, Störungen der propriozeptive Eingänge, des visuellen Systems, sowie durch internistische Erkrankungen und psychische Störungen Schwindelsymptome verursachen. Dazu zählt auch die Mitwirkung bei der Aufklärung multisensorischer und mit Stürzen assoziierter Schwindelsyndrome im höheren Lebensalter. Voraussetzung für das Verständnis sind detaillierte Kenntnisse über die Anatomie, Physiologie und Pathophysiologie und über diagnostische Verfahren.
Aufgrund der Lage des peripheren Vestibularorgans in der Otobasis stehen für das Fachgebiet der Hals-Nasen-Ohren-Heilkunde im Rahmen der Weiterbildung peripher-vestibuläre Störungen im Vordergrund ( $\bullet$ Abb. 1). Das betrifft labyrinthäre und neurogen verursachte Funktionsstörungen mit Beeinträchtigung der 5 Gleichgewichtsrezeptoren je Ohr (3 Bogengänge und die Otolithenorgane Sacculus und Utriculus) sowie des $\mathrm{N}$. vestibularis (inferior und superior) bis zum Eintritt in den Hirnstammbereich.

In den letzten Jahren haben neue Erkenntnisse über die Physiologie und Pathophysiologie vestibulärer Reflexe zu einem Paradigmenwechsel in der Differenzialdiagnostik bei „Schwindel“ geführt. Mithilfe dieser neuen Konzepte können Störungen der 5 Gleichgewichtsrezeptoren jetzt nicht nur seiten- und rezeptorspezifisch, sondern auch in einem breiten Frequenzspektrum erfasst und periphere von zentralen und nichtvestibulären Ursachen vor allem im Notfall („akutes vestibuläres Syndrom“") besser abgegrenzt werden.

Die Vieldeutigkeit des Symptoms „Schwindel“ und sein subjektiver Charakter erfordern eine sorgfältige analytische Aufarbeitung im Rahmen der Anamnese. Nur so kann die weitere differenzialdiagnostische Vorgehensweise geplant werden.

\section{Definition von „Schwindel“}

Die Bewegung des Körpers (Navigation), die Wahrnehmung der Körperposition, sowie die zeitliche und räumliche Orientierung erfordern
Abb. 1 Gleichgewichtsorgan mit 5 Rezeptoren und neurogenen Anteilen. Aus: Schünke M, Schulte E, Schumacher U. Prometheus. LernAtlas der Anatomie. Kopf, Hals und Neuroanatomie. 3. Aufl. Stuttgart: Thieme; 2012: 123.

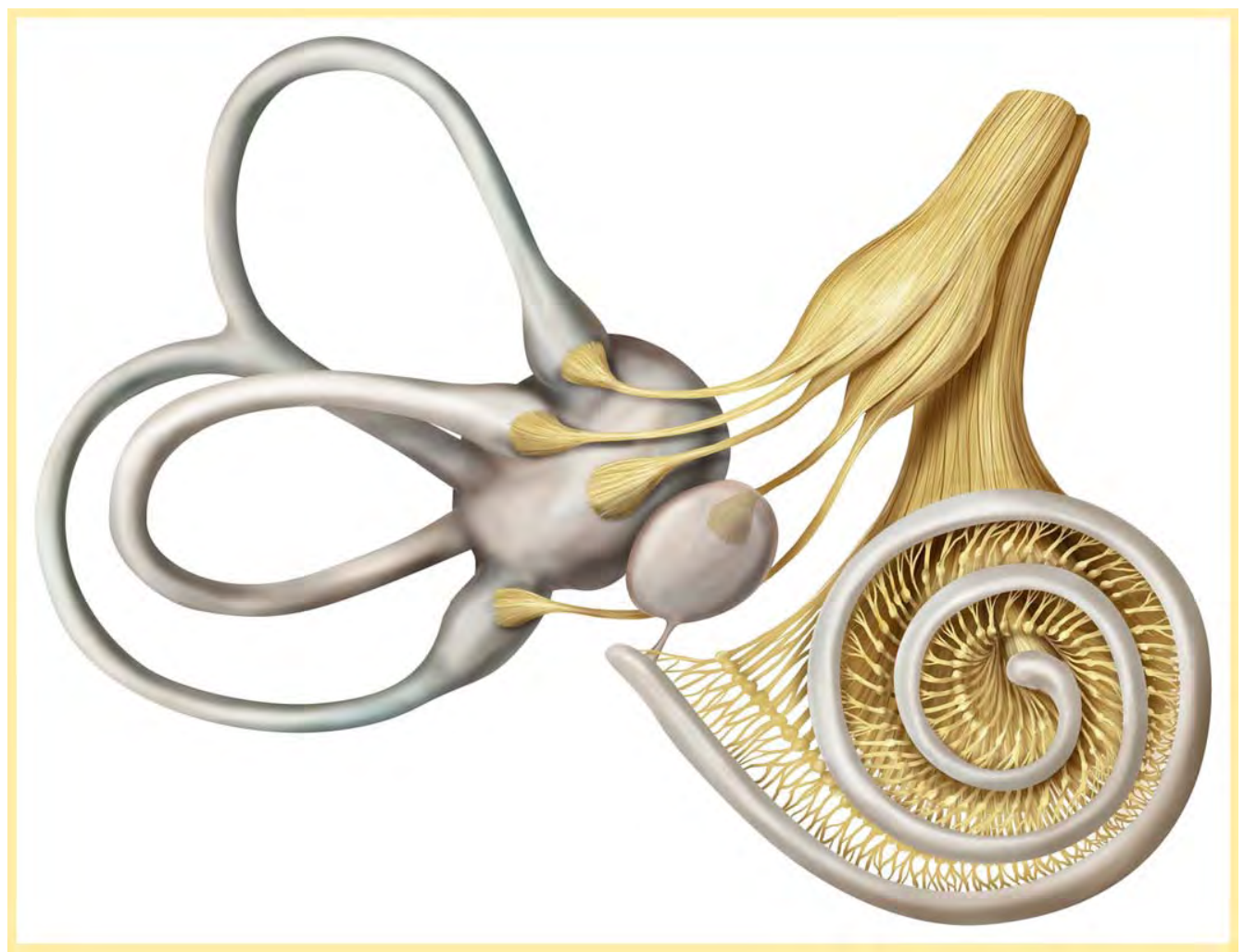


ein störungsfreies Zusammenwirken der vestibulären Sinneseingänge (5 vestibuläre Rezeptoren je Ohr), visueller (vestibulookuläre Reflexe) und propriozeptiver Informationen (vestibulospinale Reflexe) sowie ergänzender Informationen (z.B. Kreislauf, Psyche, Hörvermögen, Somatosensorik). Dieses Zusammenspiel gewährleistet eine Stabilität der Funktionen in einem breiten Dynamikbereich, sowohl in Ruhe beim Stehen und Sitzen als auch bei alltäglichen und besonderen Beanspruchungen mit schnellen Kopf- und Körperbewegungen $[1,2](\Theta$ Abb. 2$)$.

Ist das Zusammenwirken dieser verschiedenen Funktionen beeinträchtigt, entsteht „Schwindel“. Entsprechend einer aktuellen Definition nach Bisdorff et al. [3-5] unterscheidet man unter phänomenologischen Aspekten 3 unterschiedliche Definitionen von „Schwindel“:

- Das Gefühl der Eigenbewegung (Bewegungsillusionen) des Körpers wird als „Vertigo“ bezeichnet („innerer Schwindel“). Dazu zählen Empfindungen wie „Drehen“, „Schwanken“ und „Kippen“. Visuelle Bewegungsempfindungen der Umwelt (z.B. Oszillopsien) bezeichnet man als „äußerer Schwindel“.

- Eine gestörte Wahrnehmung der räumlichen Orientierung ohne Bewegungsillusionen wird als „Benommenheit“ (engl. dizziness) bezeichnet.

- Probleme beim Stehen, Gehen und im Sitzen werden als Stand- und Gangunsicherheit bezeichnet.

Merke: Unter „Schwindel“ versteht man eine gestörte Wahrnehmung der Bewegung des eigenen Körpers („innerer Schwindel“) bzw. seiner Umgebung („äußerer Schwindel“) und die Störung der räumlichen Orientierung, sowie das Gefühl der Stand- und Gangunsicherheit.

Mit dieser Definition werden alle möglichen subjektiven Empfindungen des Symptoms „Schwindel" erfasst.

„Physiologischer Schwindel“ muss von „pathologischem Schwindel“ unterschieden werden. „Physiologischer Schwindel“ stellt einen sensorischen Konflikt dar, der bei der Kombination unterschiedlicher, simultan einwirkender Reize auftritt (z. B. Höhenschwindel und Kinetosen).

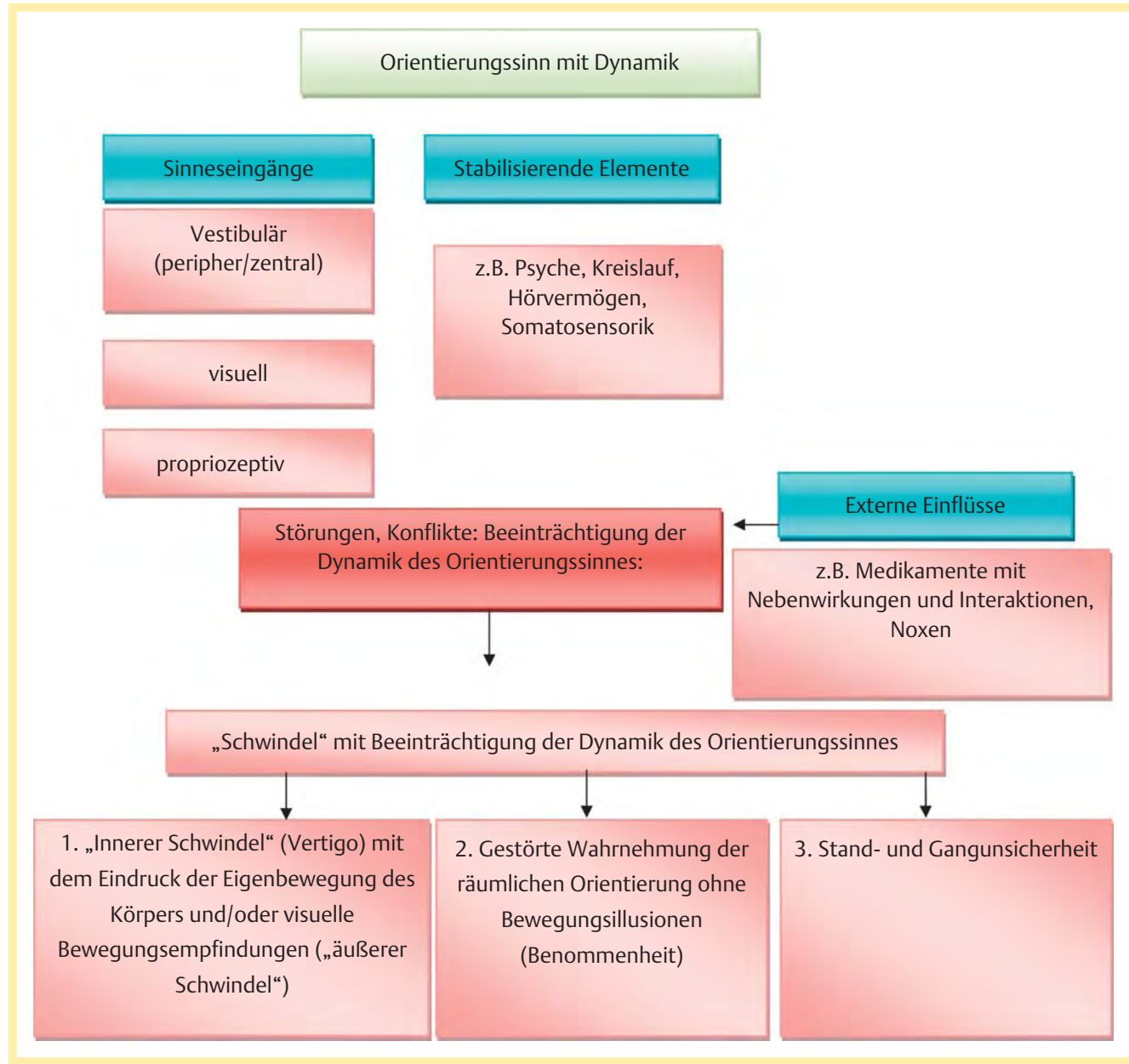

Abb. 2 Die Sinneseingänge verfügen über eine ausgedehnte dynamische Bandbreite. Damit wird die Dynamik des Orientierungssinns gewährleistet. Störungen der Sinneseingänge, der Verarbeitung der Sinneseingänge sowie äußere Einflüsse beeinträchtigen die Dynamik des Orientierungssinns und führen zu „Schwindel“. 


\section{Anamnese}

Jedem Arzt ist die Problematik bekannt, die aufgrund des subjektiven Charakters, der individuell unterschiedlichen Wahrnehmung und Interpretation sowie der Vieldeutigkeit und Ungenauigkeit des Begriffs „Schwindel“ entsteht. Patienten verstehen unter dem Symptom „Schwindel“ die unterschiedlichsten Wahrnehmungen. Diese reichen von Empfindungen wie „Drehgefühl“, „Lateropulsion“, „Liftgefühl“, „Schwanken“, „Benommenheit“ bis hin zu „Schwarz werden vor den Augen“, „Angst“ und „Leeregefühl“. Daher ist es sinnvoll, die anamnestischen Angaben zum Symptom „Schwindel“ nicht umzudeuten, sondern auch mit dem Wortlaut des Patienten zu dokumentieren, um diese Informationen sowohl im Verlauf als auch für alle anderen an der Behandlung Beteiligten im Original zur Verfügung zu haben. Daher wird im Rahmen dieser Übersichtsarbeit das Wort „Schwindel“ bewusst immer in Anführungszeichen verwendet.

Etymologisch ist das Wort „Schwindel“ aus dem althochdeutschen Verb „swintilon“ (für „In-Ohnmacht-fallen“ oder „Schwinden der Sinne“, ca. 800 n. Chr.) hervorgegangen. Erst im Mittelhochdeutschen entstand das Substantiv „Schwindel“ mit der innewohnenden Bedeutung des systematischen Schwindels. Im Rahmen der Anamnese sollte berücksichtigt werden, dass diese Bedeutung von „Schwindel“ in anderen Sprachräumen durchaus unterschiedlich aufgefasst wird.

Merke: Die Anamnese ist der Schlüssel für eine erfolgreiche und effiziente differenzialdiagnostische Abklärung des Symptoms „Schwindel“.

\section{Schwindelanalyse}

Im Rahmen der Differenzialdiagnostik hat sich die nachfolgende analytische Aufarbeitung („Schwindelanalyse“) in der Praxis bewährt [6]:

\section{A. Schwindelempfindung (subjektive Charakteristik)}

Einordnung des subjektiv empfundenen Charakters des „Schwindels“: Bewegungsillusionen des Körpers (Drehen, Schwanken) bzw. seiner Umgebung, visuelle Bewegungsempfindungen (Oszillopsien), gestörte Raumorientierung (Benommenheit), Probleme bei Stand und Gang ( $\bullet$ Abb. 3).

\section{B. Intensitäts-Zeit-Verhältnis}

Einordnung der Zeitdauer der Schwindelempfindung mit Intensität, z.B. episodischer „Schwindel" und Dauer der Attacken, permanenter (chronischer) „Schwindel“ ( $\bullet$ Abb. 3).

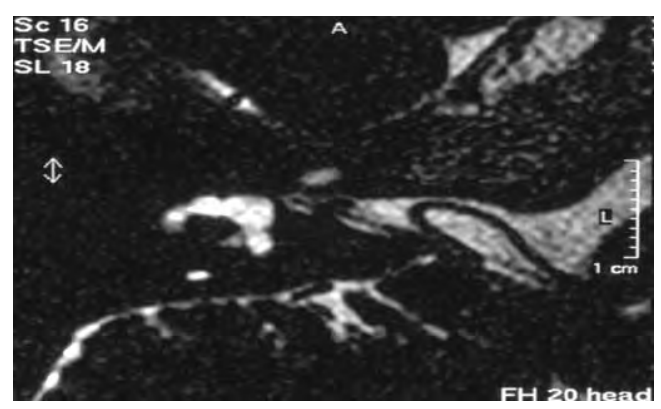

Abb. 3 Patient mit episodischem Schwindel. Anamnestisch Drehschwindelattacken von kurzer Dauer ( $<1 \mathrm{~min}$ ). Begleitsymptome sind Tinnitus und Ohrdruck. Die T2-gewichtete Kernspintomografie (CISS-Sequenz) zeigt die eher seltenere Differenzialdiagnose eines neurovaskulären Kompressionssyndroms des VIII. Hirnnerv (Vestibularisparoxysmie) durch eine Gefäßschlinge im Kleinhirnbrückenwinkelbereich links, die den N. vestibularis irritiert (roter Pfeil) (Bildquelle: Neuroradiologie Universitätsklinikum Aachen).

\section{Begleitsymptome}

Evaluierung weiterer Beschwerden bei „Schwindel“: Ohrsymptome (Ohrenschmerzen, Tinnitus, Hörstörungen, Ohrdruck, Ohrsekretion, sichtbare Hautveränderungen), Kopfschmerzen, vegetative Symptome, Bewusstseinsstörungen (Synkopen), Sturzneigung, Stürze bei erhaltenem Bewusstsein, Aura, Sehstörungen, psychische Faktoren und Begleitumstände (Angst und Panik, Depressionen, Erschöpfung, „Burn-Out“) ( $\bullet$ Abb. 4, 5).

Merke: Ohrsymptome (Ohrenschmerzen, Tinnitus, Hörstörungen, Ohrdruck, Ohrsekretion) und Schwindel weisen differenzialdiagnostisch i.d.R. auf eine peripher vestibuläre Ursache hin.

Merke: Die vestibuläre Migräne zählt zu den häufigsten Differenzialdiagosen bei episodisch auftretendem Schwindel. Die fehlenden Hörstörungen sind ein wesentliches Unterscheidungskriterium zum Morbus Menière.

\section{Provokation und Belastungsabhängigkeit} Klassifizierung von spontanem oder provozierbarem „Schwindel“. Provokation von „Schwindel" bei Lagerungswechsel oder Kopfbewegungen, bewegungsabhängige Beeinträchtigung der Sehschärfe, Schwindel bei bestimmten Tätigkeiten, z. B. im Sitzen, beim Laufen, beim Heben von Lasten, in der Höhe, visuelle Reize, Flug-, Zugund Schiffsreisen, Medikamente, Höhe, Lärm, Angstzustände bei Aufenthalt in Räumen und Hallen, Phobien ( $\bullet$ Tab. 1). 

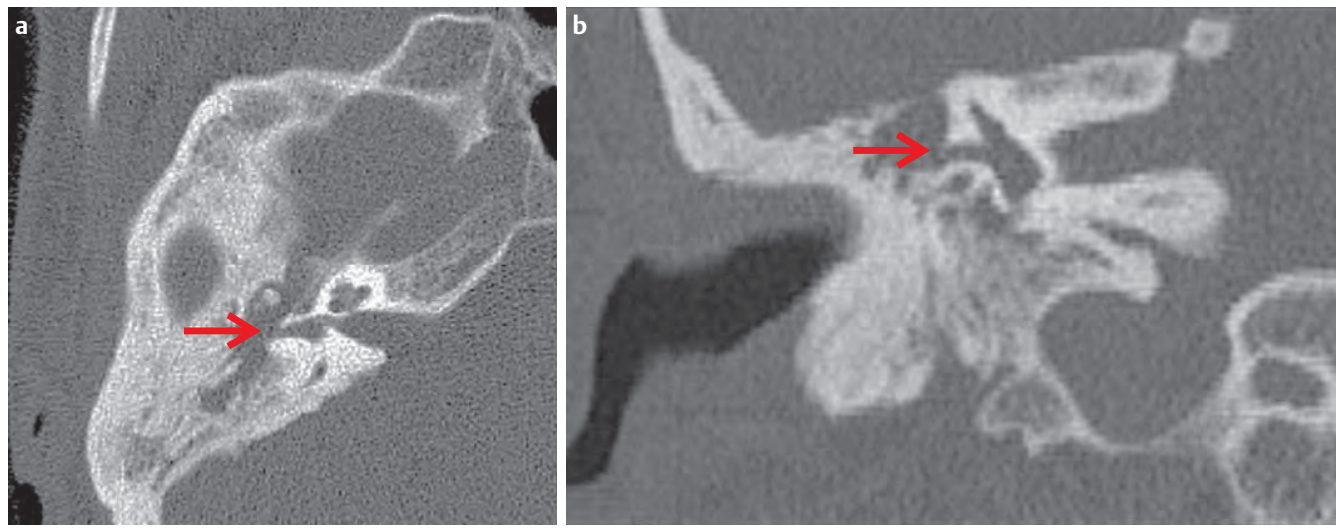

Abb. 4 a, b Schwindel bei Labyrinthfistel durch ein Cholesteatom. Die Computertomografie (a koronal, b axial) zeigt die Arrosion des rechten lateralen Bogengangs durch eine weichteildichte Masse. Wegweisend für die Diagnose waren die typischen Ohrsymptome mit rezidivierender Otorrhoe, einseitiger Hörminderung, Lateralisation nach rechts im Stimmgabelversuch nach Weber, negativer Stimmgabelversuch nach Rinne rechts, sowie ein positives Fistelsymptom (Bild: Neuroradiologie Universität Tübingen).
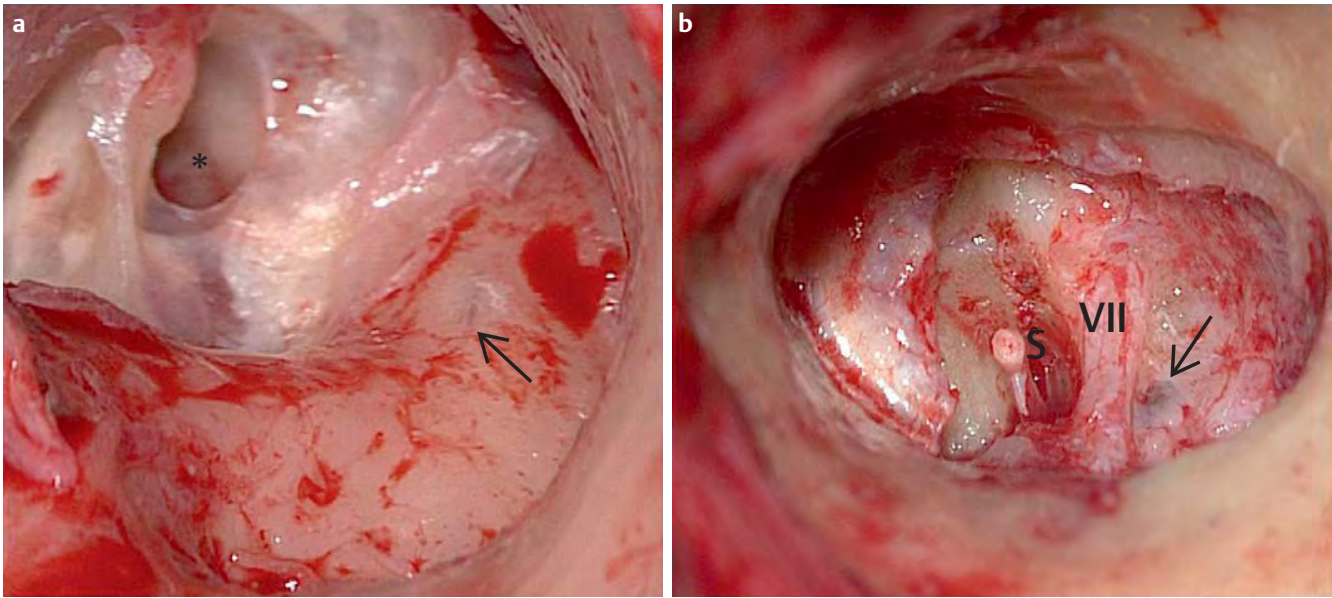

Abb. 5 a, b Die intraoperativen Bilder der Labyrinthfistel durch ein Cholesteatom zeigen eine Arrosion des linken lateralen Bogengangs a bei Zustand nach Anlage einer offenen Mastoidhöhle vor ca. 25 Jahren sowie eine Labyrinthfistel im Bereich der Einmündung des lateralen Bogengangs in das linke Vestibulum b (unterschiedliche Patienten). ${ }^{*}$ epitympanaler Defekt in der Paukenabdeckung. S: Stapes. VIl: freiliegender N. facialis links.

Tab. 1 Anamnestische Differenzierung bei episodischem Schwindel anhand der zugrunde liegenden Definitionen.

\begin{tabular}{|c|c|c|c|c|}
\hline $\begin{array}{l}\text { Benigner paroxysmaler } \\
\text { Lagerungsschwindel }\end{array}$ & Vestibuläre Migräne & Morbus Menière & Dehiszenzsyndrom & Vestibularisparoxysmie \\
\hline $\begin{array}{l}\text { - Drehschwindelatta- } \\
\text { cken< } 1 \text { min } \\
\text { - objektivierbarer VOR } \\
\text { mit kurzer Latenz und } \\
\text { Crescendo-Decrescen- } \\
\text { do meist< } 1 \text { min } \\
\text { - Augenbewegungen } \\
\text { lassen sich dem be- } \\
\text { troffenen Bogengang } \\
\text { zuordnen. } \\
\text { - Wiederkehr des } \\
\text { Nystagmus und } \\
\text { Habituation [7-9] }\end{array}$ & $\begin{array}{l}\text { - mindestens } 5 \text { Episoden mit } \\
\text { vestibulären Symptomen } \\
\text { hoher Intensität sowie Dau- } \\
\text { er von } 5 \text { min bis } 72 \mathrm{~h} \\
\text { - Kopfschmerzen während } \\
\text { der vestibulären Symptome } \\
\text { mit folgenden Kriterien: } \\
\text { Halbseitigkeit, pulsierend, } \\
\text { mäßige oder starke Inten- } \\
\text { sität, Verstärkung durch } \\
\text { gewöhnliche körperliche } \\
\text { Aktivität } \\
\text { - Foto- und Phonophobie, } \\
\text { visuelle Aura } \\
\text { - keine bessere Erklärung } \\
\text { durch eine andere Vestibu- } \\
\text { lopathie [5,10] }\end{array}$ & $\begin{array}{l}\text { - wiederkehrende } \\
\text { Drehschwindelatta- } \\
\text { cken>20 min } \\
\text { - in Kombination mit } \\
\text { (audiometrisch nachge- } \\
\text { wiesener) i.d. R. fluktuie- } \\
\text { render Hörminderung im } \\
\text { Tieftonbereich, fluktuie- } \\
\text { rendem Tinnitus sowie } \\
\text { Ohrdruck („Völlegefühl“) } \\
\text { [11] }\end{array}$ & $\begin{array}{l}\text { - mehrere Minuten andau- } \\
\text { ernde Drehschwindelat- } \\
\text { tacken mit Nystagmus } \\
\text { und Oszillopsien bei } \\
\text { akustischer Reizung und } \\
\text { pressorischen Ereignissen } \\
\text { (Tullio-Phänomen) } \\
\text { - Autophonie, Druck- und } \\
\text { Völlegefühl im Ohrbereich } \\
\text { - Hörminderung mit } \\
\text { Schalleitungsstörung im } \\
\text { niederfrequenten Bereich } \\
\text { (ab 1-2 kHz) } \\
\text { - verstärkte Wahrnehmung } \\
\text { von Knochenleitungsreizen } \\
\text { - pulsierendes Ohrgeräusch } \\
\text { [12,13,15] }\end{array}$ & $\begin{array}{l}\text { - wenige Sekunden bis } \\
\text { wenige Minuten dau- } \\
\text { ernde Dreh- oder selten } \\
\text { Schwankschwindelat- } \\
\text { tacken } \\
\text { - mit oder ohne Ohrsym- } \\
\text { ptome (Ohrdruck, Tinni- } \\
\text { tus, Hörminderung), die } \\
\text { sich häufig durch längere } \\
\text { Hyperventilation oder } \\
\text { Änderung der Kopf-Kör- } \\
\text { per-Position provozieren } \\
\text { lassen }[7,15]\end{array}$ \\
\hline
\end{tabular}

VOR: vestibulookulärer Reflex 


\section{E. Auswirkungen auf die Teilnahme am Leben in der Gesellschaft \\ Alltägliche Tätigkeiten (Treppensteigen, Radfah- ren, Autofahren).}

\section{F. Auswirkungen im Beruf}

Stehen auf Leitern, Arbeiten in der Höhe, Überkopfarbeiten, Arbeiten mit Absturzgefahr, Fahren auf vibrierenden Maschinen, Arbeit an laufenden Maschinen, Arbeiten in Gefahrenbereichen.

\section{G. Ergänzende Angaben}

Vorerkrankungen, Medikamenten- und Noxeneinnahme, stattgehabte Unfälle (z.B. Kopfanpralltraumen, Schädelfrakturen), Applikation otoxischer Medikamente, Ohrerkrankungen, erfolgte Operationen am Ohr, Zeckenstiche, Infektionskrankheiten, Auslandsaufenthalt, Vorgeschichte akuter Hör- und Gleichgewichtserkrankungen, Stürze und Beinahe-Stürze, ggf. mit Verletzungsfolge.

\section{Vor- und Begleiterkrankungen sowie Medikamenteneinnahme}

Die „Schwindel“-Anamnese sollte auch Vor- und Begleiterkrankungen und eine Medikamentenanamnese umfassen:

\section{Chronischer Schwindel}

Patienten mit einem länger anhaltenden (chronischen) Schwindel, der über das somatisch Erklärbare hinausgeht oder ohne fassbare funktionelle

Vor-, Begleiterkrankungen und Medikamentenanamnese

- neurologische Erkrankungen: Apoplex, andere zerebrale Ischämien, Migräne, HOPS, ZNS-Neubildungen, Epilepsie

- kardiovaskuläre Erkrankungen („mit zerebraler Hypoxie“): HRS, HI, Vitien, Gefäßstenosen (Operationen?), persistierendes Foramen ovale, aHTN

- endokrinologische Erkrankungen: Diabetes mellitus, Hypo-/Hyperthyreose

- ophthalmologische Erkrankungen: Visusund Gesichtsfeldveränderungen, Strabismus, Glaukom, „neue/alte Brille“

- psychiatrische/psychosomatische Erkrankungen

- Sonstiges: Anämie, Tumorerkrankungen, Erkrankungen des muskuloskelettalen Systems (einschließlich Operationen an Wirbelsäule und Gelenken), kraniomandibuläre Dysfunktion

- Medikamentenanamnese (einschließlich Wechselwirkungen): Herz-Kreislauf-Medikation, Sedativa/Schlafmedikation, Neuroleptika und andere Psychopharmaka, Schilddrüsenmedikation
Beeinträchtigung stellen für Untersucher und Behandler eine vielfältige Herausforderung dar [7,16-18].

$\mathrm{Zu}$ den chronischen subjektiven Schwindelformen (Schwindel $>3$ Monate, der alle genannten Kriterien der Definition „Schwindel“ betreffen kann) zählen somatoforme Schwindelformen $[7,16,17]$. Wenn keine vorherige organische Schwindelerkrankung vorhanden ist (z. B. bei zugrunde liegender Angstsymptomatik, Panik, Depression), spricht man von einem primären somatoformen Schwindel. Ein sogenannter sekundärer somatoformer Schwindel kann sich z. B. bei Patienten mit episodischen, rezidivierenden, die Lebensqualität stark beeinträchtigenden chronischen Vestibulopathien (benigner paroxysmaler Lagerungsschwindel, vestibuläre Migräne, Morbus Menière), nichtkompensierten chronischen Vestibulopathien (Neuritis vestibularis, Kleinhirnerkrankungen) und nichtvestibulär bedingten Störungen mit Synkopen usw. entwickeln. Typisch sind eine Persistenz von Schwindelsyndromen über mehrere Wochen mit einer Diskrepanz zwischen dem natürlichen Verlauf einer Erkrankung, der Ausprägung der krankheitsspezifischen Symptomatik und den erhobenen Befunden [7,16,17].

Merke: Chronische Schwindelsyndrome (chronischer subjektiver Schwindel) ohne oder infolge einer organischen Erkrankung sind häufig und müssen in die differenzialdiagnostischen Überlegungen einbezogen werden.

\section{Schwindelsyndrome und Lebensalter $\nabla$}

Auch das Alter des Patienten erlaubt im Rahmen der Anamnese im Hinblick auf die altersspezifische Häufigkeitsverteilung von Schwindelsyndromen Rückschlüsse auf mögliche Differenzialdiagnosen. „Schwindel“ kann in jedem Lebensalter auftreten. Im höheren Lebensalter gehört „Schwindel“ zu den häufigsten Beschwerden. Die diagnostische Konstellation von Schwindelsyndromen im Kindes- und Jugendalter unterscheidet sich von der im höheren Lebensalter (० Tab. 2) $[19,20]$.

Die vestibuläre Migräne gehört zu den häufigsten Erkrankungen mit „Schwindel“ im Kindes- und Jugendalter. Bei Hochbetagten dominiert der benigne paroxysmale Lagerungsschwindel. Ein multisensorischer "Schwindel“ ist eine Beeinträchtigung von mehreren (mindestens 2) Bestandteilen des Orientierungssinns [1]. Er kommt im höheren Lebensalter häufig vor. 
Tab. 2 Häufige Erkrankungen mit „Schwindel“ im Kindes- und Jugendalter sowie im höheren Lebensalter.

Schwindelsyndrome im Kindes- und Jugendalter

- vestibuläre Migräne

- psychisch bedingter Schwindel

- orthostatische Dysregulation
Schwindelsyndrome im höheren Lebensalter

- benigner paroxysmaler Lagerungsschwindel

- psychisch bedingter Schwindel

- multisensorischer „Schwindel“

- Herzrhythmusstörungen
Merke: Die vestibuläre Migräne gehört zu den häufigsten Erkrankungen mit „Schwindel“ im Kindes- und Jugendalter. Bei Hochbetagten dominiert der benigne paroxysmale Lagerungsschwindel.

\section{Anamnese bei „Schwindel“ und Stürzen im höheren Lebensalter}

Stürze und Ihre Folgen bedeuten für den Betroffenen einen Verlust an Lebensqualität, Unabhängigkeit und Selbstbestimmung. „Schwindel“ und Stürze können einen Kreislauf verursachen, der zu Inaktivität (durch Sturzangst), häuslicher Isolation, zu einem Verlust an sozialen Kontakten und zu depressiven Entwicklungen führen kann. Bei etwa jedem zehnten Sturz sind schwerwiegende Verletzungen mit potenzieller Pflegebedürftigkeit die Folge.

Prinzipiell ist jeder Patient mit Schwindel und Gleichgewichtsstörungen sturzgefährdet. Im höheren Lebensalter sind krankheitsbedingte Stürze aufgrund multisensorischer Defizite und Komorbiditäten häufiger. Physiologische Veränderungen im Bereich der sensorischen Elemente des Orientierungssinns (z.B. Degeneration von Otokonien, Verminderung der Anzahl der Rezeptoren im Innenohr, zentrale vestibuläre Veränderungen, Reduktion der Haut- und Gelenkrezeptoren sowie der intrafusalen Fasern der Muskelspindeln, Beeinträchtigung der Flexibilität der Augenlinse usw.) führen zu einer Verminderung des Dynamikbereichs des Orientierungssinns im höheren Lebensalter $[2,21]$.

Kommen zu diesen physiologischen Veränderungen pathologische Veränderungen der sensorischen Elemente (Neuritis vestibularis, Otolithenfunktionsstörungen), der stabilisierenden Elemente (z.B. Hörvermögen) oder Begleiterkrankungen mit unvermeidlichen externen Einflüssen (Medikamentenapplikationen und deren Nebenwirkungen und Interaktionen) hinzu, erhöht sich die Wahrscheinlichkeit von Stürzen um ein Vielfaches.

Aufgrund demografischer Veränderungen in den Industriestaaten wird die Altersgruppe der Senioren in den kommenden Jahren prozentual zunehmen. Daher muss sich der HNO-Facharzt künftig intensiver mit dem Problem von „Schwindel“ und Stürzen auseinandersetzen.
Merke: „Schwindel“ im höheren Alter stellt einen Sturzrisikofaktor dar.

Die „Sturz-Anamnese“ erfasst mit 12 Fragen die wichtigsten Sturzrisikofaktoren wie „Schwindel“, Sehstörungen, muskuloskelettale Veränderungen, psychische Beeinträchtigung und Hörvermögen [21].

Vor allem hinsichtlich der aktuellen Medikamenteneinnahme sollte die Anamnese bei „Schwindel“ besonders sorgfältig erhoben werden. „Schwindel“ kann als Nebenwirkung bei der Einnahme von psychotropen Pharmaka wie Benzodiazepin-Derivaten, Antidepressiva und Neuroleptika entstehen. Auch Klasse-1 A-Antiarrythmika und hypotensiv wirksame Medikamente sind mit einem hohen Sturzrisiko behaftet. Neben diesen können Diuretika vor allem im Rahmen von Interaktionen zu Nebenwirkungen mit Sturzfolge führen.

\section{Sturzanamnese durch Ermittlung der}

Sturzrisikofaktoren (nach Walther 2012 [21])

- Leiden Sie an Muskelschwäche?

- Sind Sie im letzten Jahr oder früher schon einmal gestürzt?

- Haben Sie Probleme beim Gehen/Laufen?

- Leiden Sie an Schwindel/Gleichgewichtsstörungen?

- Nutzen Sie Hilfsmittel zur Fortbewegung?

- Ist Ihr Sehvermögen beeinträchtigt?

- Leiden Sie an Gelenkbeschwerden der Beine/ Hüfte?

- Sind Sie in Ihren täglichen Aktivitäten eingeschränkt?

- Leiden Sie unter Depressionen?

- Ist Ihr Erinnerungs-/Merkvermögen beeinträchtigt?

- Nehmen Sie mehr als 5 verschiedene Medikamente?

- Sind sie schwerhörig?

Bei mehr als 3 Risikofaktoren ist mit einem erhöhten Sturzrisiko zu rechnen [21].

Merke: Die Evaluierung der Medikation einschließlich der Wechselwirkungen ist vor allem bei "Schwindel“ im höheren Lebensalter mit drohenden und stattgefundenen Stürzen von Bedeutung. 


\section{Anamnese bei „Schwindel“ und Synkopen \\ $\nabla$}

Von wichtiger differenzialdiagnostischer Bedeutung bei „Schwindel“ ist die Frage, ob bei Patienten mit Sturz- und Fallneigung eine Störung des Bewusstseins beteiligt ist $[22,23]$.

Unter einer Synkope wird eine plötzlich einsetzende, kurz andauernde Bewusstlosigkeit verstanden, die mit einem Verlust der Haltungskontrolle einhergeht und spontan wieder sistiert. Differenzialdiagnostisch muss ein „Hinstürzen“ abgegrenzt werden, bei dem das Bewusstsein erhalten bleibt. Hierzu zählen vor allem lokomotorisch bedingte Stürze im höheren Lebensalter. Bei der sogenannten Tumarkin-Otolithenkrise (,vestibular drop attacks“) wird eine Dysfunktion des Sacculus angenommen ( $\bullet$ Tab. 3, 4) [22-24].

Cave: Bei „Schwindel“ im höheren Lebensalter treten kardiovaskulär bedingte Störungen häufiger auf. Findet sich bei episodischem Schwindel bei Senioren keine vestibuläre Ursache, muss differenzialdiagnostisch vor allem an eine kardiovaskuläre Erkrankung gedacht werden.

\section{Validierte Fragebögen zur Ergänzung der Schwindel-Anamnese \\ $\nabla$}

Ergänzend lässt sich die individuelle subjektive Beeinträchtigung durch „Schwindel“ mit strukturierten und validierten Testverfahren bewerten [25-27]. Das Dizziness Handicap Inventory (DHI) steht als validierte Versionen auch in deutscher Sprache zur Verfügung. Diese Evaluierung kann ergänzend zu den anamnestischen Angaben ggf. zur Differenzierung physischer, funktioneller sowie emotionaler Beeinträchtigungen beitragen. Je nach führender Symptomatik und Problematik (z.B. Stürze) können weitere evaluierte Fragebögen zum Einsatz kommen, z. B. Hospital Anxiety Subscale (HADS-A), Activities-Specific Balance Confidence (ABC) Scale oder Activities of Daily Living (ADL) zur Erfassung grundlegender Alltagsfunktionen [22,23].
Merke: Die Anamnese ist der Schlüssel zur gezielten und effektiven Initialdiagnostik von Patienten mit Schwindel. Sie umfasst die Beschreibung der Symptome (auch dokumentieren mit den Worten des Patienten) nach Charakteristik, Intensität, Beginn und Zeitdauer, Auslösbarkeit (Provozierbarkeit), Belastungsabhängigkeit, Auswirkungen auf die Teilnahme am täglichen Leben und im Beruf sowie Fragen nach Begleitsymptomen, Vor- und Nebenerkrankungen (z. B. otologisch, traumatologisch, neurologisch, kardiovaskulär, endokrinologisch, muskuloskelettal, ophthalmologisch, psychiatrisch/psychosomatisch) und Medikamenten. Grundsätzlich sollte die Anamnese sowohl eine offene Frage am Anfang beinhalten („Wie würden Sie den Schwindel beschreiben?“), die dem Patienten eine freie Schilderung erlaubt, als auch geschlossene Fragen („Dauer- oder Attackenschwindel?“), anhand derer der Arzt dann konkrete Differenzialdiagnosen evaluiert.

Die Aufarbeitung aller Informationen aus der Anamnese trägt dazu bei, vestibuläre (peripher vestibuläre und/oder zentral bedingte Störungen) von nichtvestibulären Störungen zu differenzieren, und liefert wichtige Informationen für die Planung der weiteren, sich an die Anamnese anschließenden Diagnostik.

\section{Screening-Untersuchungen in der Differenzialdiagnostik}

\section{Augenbewegungsstörungen und Nystagmus}

Störungen der Augenbewegungen und Nystagmus finden sich bei HNO-ärztlichen, neurologischen und ophthalmologischen Erkrankungen. Neben „Schwindel“ klagen die betroffenen Patienten häufig über „Augensymptome“, wie eine Beeinträchtigung der Sehschärfe („Verschwommensehen“), Doppelbilder oder laufende Bilder (Oszillopsien).

Der Untersuchungsgang beginnt mit einer Inspektion. Dabei sollten u.a. die Relation von Kopfund Körperhaltung, die Stellung der Augen zueinander und ggf. sichtbare spontane Augenbewegungen berücksichtigt werden. Störungen der Kopfhaltung treten z.B. bei Augenmuskelparesen

Tab. 3 Mögliche Ursachen bei „Schwindel“ im Zusammenhang mit Synkopen/Bewusstseinsstörungen.

\begin{tabular}{|c|c|c|c|}
\hline Zirkulatorisch & Metabolisch, medikamentös & $\begin{array}{l}\text { Zerebrovaskulär, neuro- } \\
\text { logisch, psychiatrisch }\end{array}$ & Kardiogen \\
\hline $\begin{array}{l}\text { - orthostatische Dysre- } \\
\text { gulation } \\
\text { - vasovagale Synkope } \\
\text { - hypersensitiver } \\
\text { Karotissinus }\end{array}$ & $\begin{array}{l}\text { - Hypoglykämie } \\
\text { - Schilddrüsenüberfunktion } \\
\text { (thyreotoxische Krise) } \\
\text { - Medikamentenüberdosie- } \\
\text { rung }\end{array}$ & $\begin{array}{l}\text { - Subclavian-Steal- } \\
\text { Syndrom } \\
\text { - Schlaganfall } \\
\text { - Basilaristhrombose } \\
\text { - Panikattacken }\end{array}$ & $\begin{array}{l}\text { - Herzrhythmusstörungen } \\
\text { - Lungenembolie } \\
\text { - Aortenstenose }\end{array}$ \\
\hline
\end{tabular}


Tab. 4 Differenzialdiagnose des Schwindels. Die Anamnese ist der Schlüssel zur gezielten und effektiven Differenzialdiagnose bei der Initialdiagnostik von Patienten mit Schwindel! Sie umfasst die Beschreibung der Symptome (auch dokumentieren mit den Worten des Patienten) nach Charakteristik, Intensität, Beginn und Zeitdauer, Auslösbarkeit (Provozierbarkeit), Belastungsabhängigkeit, Auswirkungen auf die Teilnahme am täglichen Leben und im Beruf sowie Fragen nach Begleitsymptomen, Vor- und Nebenerkrankungen (z. B. otologisch, traumatologisch, neurologisch, kardiovaskulär, endokrinologisch, muskuloskeletal, ophthalmologisch, psychiatrisch/psychosomatisch) und Medikamenten.

\begin{tabular}{|c|c|c|c|c|}
\hline & Beginn - Dauer & & & Charakteristika \\
\hline & akut - anfallsartig & akut - andauernd & allmählich - andauernd & \\
\hline $\begin{array}{l}\text { peripher } \\
\text { vestibulär }\end{array}$ & $\begin{array}{l}\text { - benigner paroxysmaler } \\
\text { Lagerungsschwindel } \\
\text { - Morbus Menière } \\
\text { - Tumarkin-Krise } \\
\text { - Tullio-Phänomen }\end{array}$ & $\begin{array}{l}\text { - Neuritis vestibularis } \\
\text { - Felsenbeinfraktur } \\
\text { - Contusio labyrinthi } \\
\text { - Zoster oticus } \\
\text { - Labyrinthitis } \\
\text { - Cholesteatom }\end{array}$ & $\begin{array}{l}\text { - „Multisensorischer } \\
\text { Schwindel“ }\end{array}$ & $\begin{array}{l}\text { - systematisch, Richtungstendenz } \\
\text { - Drehschwindel, Lateropulsion, } \\
\text { Liftgefühl, Fallgefühl, Lagerungs- } \\
\text { schwindel }\end{array}$ \\
\hline zentral & $\begin{array}{l}\text { - Basilarisinsuffizienz } \\
\text { - Migräne-assoziierter Schwindel } \\
\text { - mikrovaskuläre Kompressions- } \\
\text { syndrome } \\
\text { - (Epilepsie) }\end{array}$ & $\begin{array}{l}\text { - Schlaganfall } \\
\text { - Neubildungen }\end{array}$ & $\begin{array}{l}\text { - hirnorganische Syndrome } \\
\text { - zerebrale Durchblutungs- } \\
\text { störungen } \\
\text { - Medikamentenintoxi- } \\
\text { kation }\end{array}$ & $\begin{array}{l}\text { - unsystematisch } \\
\text { - Unsicherheitsgefühl, Schwank- } \\
\text { schwindel, Benommenheitsgefühl, } \\
\text { „Entrücktsein“ }\end{array}$ \\
\hline $\begin{array}{l}\text { nichtvesti- } \\
\text { bulär }\end{array}$ & $\begin{array}{l}\text { - internistische Erkrankungen } \\
\text { Herzinsuffizienz, Vitien, Gefäß- } \\
\text { stenosen }\end{array}$ & - & $\begin{array}{l}\text { - Anämie } \\
\text { - endokrinologische } \\
\text { Erkrankungen } \\
\text { - ophthalmologische } \\
\text { Erkrankungen (Glaukom, } \\
\text { Myopie, Strabismus) }\end{array}$ & $\begin{array}{l}\text { - unsystematisch } \\
\text { - Schwarzwerden vor den Augen, } \\
\text { Sternchensehen, Verschwommen- } \\
\text { sehen, Kollaps, Leeregefühl, Unsi- } \\
\text { cherheitsgefühl, Schwankschwindel }\end{array}$ \\
\hline psychogen & $\begin{array}{l}\text { - psychogener Schwindel in } \\
\text { Kombination mit psychischen } \\
\text { Symptomen wie Angst }\end{array}$ & & & $\begin{array}{l}\text { - unsystematisch } \\
\text { - Dominanz vegetativer Symptome, } \\
\text { Schwächegefühl, Angst, auch } \\
\text { Dauerschwankschwindel oder } \\
\text { Benommenheit }\end{array}$ \\
\hline
\end{tabular}

Tab. 5 Merkmale des peripher und des zentral bedingten Spontannystagmus (für Details siehe [7]).

\section{Peripher bedingter Spontannystagmus}

- horizontal rotierend (auch bei zentraler Störung [Kleinhirn] möglich)

- meist zum gesunden Ohr (bei Reiznystagmus, z. B. Morbus Menière, zum kranken Ohr)

- geht mit Fallneigung und Schwindelgefühl einher

- verringert sich im Laufe der vestibulären Kompensation

\section{Zentral bedingter Spontannystagmus}

- horizontal oder vertikal rotierend

- ist nicht durch Fixation unterdrückbar (ggf. Intensitätszunahme bei Fixation)

- seltener von Schwindelsymptomen begleitet
(z.B. M. trochlearis) oder einer „ocular tilt reaction“ (Symptomkomplex mit Abweichung der beiden Augen in der Vertikalebene, Verkippung der subjektiven Vertikalen und ipsiversive Kopfneigung) auf. Zur Differenzialdiagnose von vertikalen Fehlstellungen der Augenachsen („Skew deviation“) nutzt man den Abdeck-Test [7,28-30].

Merke: Bei peripheren Augenbewegungsstörungen ist bis auf wenige Ausnahmen (z. B. Myasthenia gravis) nur ein Auge betroffen. Zentrale Augenbewegungsstörungen betreffen in der Regel beide Augen.

Im Rahmen der klinischen Untersuchung muss weiterhin die Augenstellung in den 9 Blickpositionen untersucht werden. In der Primärposition kann ein Spontannystagmus richtungsabhängig horizontal, vertikal oder rotierend auftreten. Horizontale Spontannystagmen ggf. mit rotierender Komponente sind meist peripher bedingt, können aber auch durch eine zentrale Störung des vestibulookulären Reflexes (VOR) induziert werden. Ein ausschließlich vertikaler Spontannystag- mus hat eine zentrale Ursache (siehe Video 1 online). Lässt sich ein horizontal-rotierender Spontannystagmus (siehe Video 2 online) durch visuelle Fixation eines stationären Zieles unterdrücken, handelt es sich meist um einen peripheren vestibulären Spontannystagmus (z.B. bei einer peripheren Vestibulopathie). Ist das nicht der Fall, bzw. nimmt ein Nystagmus bei Fixation zu, liegt eine zentrale Störung („zentraler Fixationsnystagmus“) vor. Bei der Untersuchung der Blickpositionen lassen sich auch Blickhaltedefekte (z.B. Endstellnystagmus, Blickrichtungsnystagmus) erkennen [7,28-30].

Cave: Ein Spontannystagmus ist eine objektives Zeichen einer Störung des VOR. Er kann somit peripher und/oder auch zentral vestibulär bedingt sein ( $\bullet$ Tab. 5). Zentrale Störungen (Hirnstamm-, Kleinhirninfarkte) können eine nahezu identische Befundkonstellation wie periphere Störungen aufweisen und sind in der Lage, das Bild einer peripheren Störung zu „imitieren“ [31-33]. 
Abb. 6 Akutes vestibuläres Syndrom bei Kleinhirninfarkt. Der Patient zeigte vordergründig klinische Symptome einer peripheren Vestibulopathie.

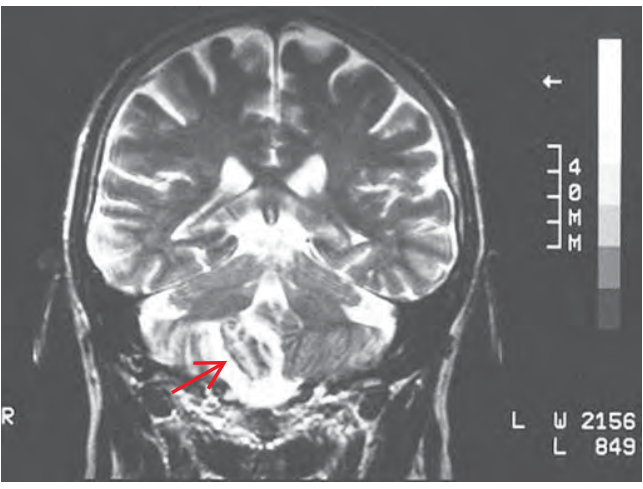

Ein Endstellnystagmus ist pathologisch, wenn dieser mehr als 5 Schläge oder $>20$ s anhält. Er ist häufig medikamenteninduziert (Antiepileptika, Benzodiazepine) oder toxisch verursacht (Alkohol). Blickrichtungsnystagmen lassen sich anhand ihrer Formen grob topografisch-anatomisch zuordnen - bspw. horizontale (z.B. bei Läsionen im Vestibulariskerngebiet), vertikale (z.B. bei Mittelhirnläsionen) und allseitige Blickrichtungsnystagmen (z.B. bei Kleinhirnläsionen) [7,28-30] ( $\odot$ Abb. 6).

Merke: Findet sich bei „Schwindel“ ein Blickrichtungsnystagmus, d.h. ein horizontaler, mit der Blickrichtung richtungswechselnder Nystagmus, macht dies eine zentrale Augenbewegungsstörung sehr wahrscheinlich.

Zentrale Augenbewegungsstörungen können auch die langsamen Augenfolgebewegungen (z.B. sakkadierte langsame Blickfolge, z.B. bei zentralen Okulomotorikstörungen) und das Sakkadensystem beeinträchtigen (Hypometrien, Hypermetrien, Beeinträchtigung der Zielgenauigkeit z. B. bei einer internukleären Ophthalmoplegie, Kleinhirnstörungen usw.). Bei der Einschätzung kann davon ausgegangen werden, dass horizontale Augenbewegungen im Pons generiert und gesteuert werden, vertikale und rotierende Augenbewegungen im Mesenzephalon (siehe Video 1 und 3 online).

Optokinetische Untersuchungen (z.B. mit einer Streifentrommel) erfordern ein intaktes Sehvermögen und prüfen die Mittelhirn- und Ponsregionen. Besteht der Verdacht auf eine periphere und zentrale Augenbewegungsstörung oder ei-

Differenzialdiagnostische klinische Zeichen einer zentral bedingten vestibulären Störung [7]

- deutliche Diskrepanz zwischen Nystagmusintensität und dem subjektiven Schwindelgefühl

- unsystematischer Schwindel

vertikaler oder rein torsioneller Nystagmus
Störungen der langsamen Blickmotorik (sakkadierte langsame Augenfolgebewegungen)

- Blickrichtungs- oder regelloser Nystagmus

- Lagennystagmus von über 2-minütiger Dauer

- fehlende Fixationssuppression

- kleine dysrhythmische, dysmetrische Nystagmusschrift

- Kombination mit anderen neurologischen Zeichen (Kopfschmerzen, Sehstörungen)

nen Nystagmus, der zentral generiert wird, muss eine augenärztliche und neurologische Differenzialdiagnostik erfolgen.

\section{Untersuchung mit der Frenzel-Brille und Provokationsnystagmus \\ $\nabla$}

Die Untersuchungen mit Frenzel-Brille in Primärposition (Geradeausblick) und eines Provokationsnystagmus sind dann hilfreich, wenn sich bei der visuellen, nichtapparativen Diagnostik kein Hinweis für Augenbewegungsstörungen und Nystagmus findet.

Die Provokation durch Kopfschütteln (Provokationsnystagmus, „head shaking nystagmus“) in der Ebene des horizontalen Bogengangs stellt ein sensitives Verfahren dar, um eine einseitige periphere Vestibulopathie zu demaskieren. Der durch Kopfschütteln (10-15× beidseits, ca. $2 \mathrm{~Hz}$ ) induzierte horizontale Nystagmus (vestibulookulärer Reflex, VOR) schlägt in den meisten Fällen mit seiner langsamen Phase in Richtung der Seite der Störung (siehe $\bigcirc$ Video 4 online). Dieser Provokationsnystagmus ist ein Hinweis auf eine Asymmetrie des „Geschwindigkeitsspeichers“ („velocity storage“) im Hirnstamm. Differenzialdiagnostisch ist ein Kopfschüttelnystagmus auch bei zentralen Störungen nachzuweisen.

Zeigt sich bei Provokation ein vertikaler Nystagmus, spricht man von einem widernatürlichen, atypischen Nystagmus („perverted head shaking nystagmus“), der bei fokalen pontinen Infarkten, aber auch bei Patienten mit einer vestibulären Migräne beschrieben ist [7,30,34].

\section{Lage- und Lagerungsschwindel}

Besteht der Verdacht auf einen lage- oder lagerungsabhängigen Nystagmus (z. B. benigner paroxysmaler Lagerungsschwindel, „benign paroxysmal positional vertigo“, BPPV), muss eine adäquate Provokation erfolgen.

Differenzialdiagnostisch ist ein seltener (zentraler) Lagenystagmus zu berücksichtigen, der nach der Lageänderung länger ( $>2 \mathrm{~min}$ ) anhält, aber 
prinzipiell eine ähnliche Symptomatik wie der BPPV aufweist $[7,8,35,36]$.

\section{Untersuchung von Stand und Gang \\ $\nabla$}

Vestibuläre, visuelle und propriozeptive Störungen der Sinneseingänge wirken sich auf die Standstabilität aus. Mit dem Romberg-Versuch und seinen Varianten kann die Balance unter statischen Bedingungen geprüft werden. Bei einseitigen peripheren Vestibulopathien tritt eine Fallneigung in Richtung des gestörten Ohres auf. Somit ändert sich bei Kopfdrehung (z.B. um $90^{\circ}$ ) die Richtung der Fallneigung (nach hinten).

Bei multisensorischen Störungen im höheren Lebensalter kann eine vermehrte Instabilität beim Schließen der Augen beobachtet werden. Psychogene Störungen sind oft durch ein regelloses Schwanken mit drohender Fallneigung gekennzeichnet. Bei zentralen Störungen ist eine Fallneigung nach vorn und hinten häufig $[7,15,35]$. Entsprechend evidenzbasierter Kriterien kann ein Sturzrisiko-Check in kürzerer Zeit mittels Aufsteh- und Gehtest oder dem Geh- und Zähltest erfolgen. Aufwändiger ist der Tinetti-Test, bei dem Gang und Gleichgewichtsfaktoren in einen Score eingehen, der das Sturzrisiko numerisch ausdrückt [21-23].

\section{Untersuchung des vestibulookulären Reflexes (VOR)}

Der VOR ist ein wesentlicher Reflex des vestibulären Systems, da er unter anderem die Blickstabilisierung bei Körper- und Kopfbewegungen im Hochfrequenzbereich realisiert. Er verbindet die vestibulären Sinneseingänge (Rezeptoren der Bogengänge) über zentrale Verschaltungen (,3-Neuronen-Reflexbogen“: N. trochlearis, N. oculomotorius, N. abducens) mit den Augenmuskeln. Die Latenzzeit des VOR ist extrem kurz $(<10 \mathrm{~ms})$. Betrachtete stationäre Objekte werden bei allen Körper- und Kopfbewegungen simultan auf gleichen Orten der Netzhaut abgebildet. Kopf- und Augenbewegung laufen daher bei ungestörter Blickstabilisierung nahezu verzögerungslos ab [7].

Der hochfrequente Anteil des VOR der Bogengänge wird mit dem klinischen Kopfimpulstest (KIT) nach Halmagyi und Curthoys geprüft (siehe Video 13 online) [37]. Störungen des VOR können labyrinthär bedingt sein oder im Bereich der hochfrequenten Nervenfasern lokalisiert sein. Sie äußern sich dann in einer Dissoziation von Kopfund Augenbewegung, die als Rückstell- bzw. Korrektursakkaden sichtbar werden [37].
Merke: Der Kopfimpulstest dient der Untersuchung des vestibulookulären Reflexes (VOR). Bei intakter Funktion zeigt sich bei impulsartiger Kopfdrehung (ca. $15^{\circ}$ ) in der Ebene des geprüften Bogengangs zur gesunden Seite bei gleichzeitiger Fixation eines unbeweglichen Zieles ein stabiler Blick. Bei einer Funktionsstörung des VOR (Störung der Blickstabilisierung) ist nach (oder noch während), der impulsartigen Kopfdrehung zur erkrankten Seite bei gleichzeitiger Fixation eines unbeweglichen Zieles eine kompensatorische Korrektursakkade zur kontralateralen Seite sichtbar, die wir als „3-K-Regel“ bezeichnen.

Wie beim gutartigen Lagerungsschwindel entspricht die Richtung der induzierten Korrektursakkade einer Augendrehachse, die senkrecht zur Ebene des impulsartig erregten Rezeptors der Bogengänge liegt. Findet sich eine Störung des VOR (sichtbare Korrektursakkaden), dann liegt aus dynamischer Sicht eine Beeinträchtigung des höheren Frequenzbereichs (ca. 4-7Hz) des VOR vor. Es muss berücksichtigt werden, dass es auch einen niederfrequente Anteil des hVOR gibt, der mit der thermischen Prüfung $(<0,01 \mathrm{~Hz})$ erfasst wird. Beide Frequenzbereiche können auch unabhängig voneinander beeinträchtigt sein [2,37-39]. Der klinische Kopfimpulstest ist eine obligate Untersuchung bei „Schwindel“. Ein Spontannystagmus stört die Untersuchung nicht. Eine apparative Visualisierung des VOR erfolgt mit dem Videokopfimpulstest, der in unterschiedlichen technischen Versionen erstmals von Ulmer \& Chays 2005, Weber et al. 2009, Bartl et al. 2009 und MacDougall et al. 2013 beschrieben worden ist [40-43] (॰ Abb. 7).

Bei einer Störung des VOR ist die Geschwindigkeit der Augenbewegung vermindert (Gain $<0,8$ ). Es kommt zur Ausbildung von reflektorischen Korrektursakkaden. Diese Korrektursakkaden können im klinischen Kopfimpulstest sichtbar sein ( 0 Abb. 8). Eine Visualisierung der Störungen des VOR ist z.B. mit der App „aVOR“ von Liberty IT, Crows Nest, Australien, möglich.

Liegt eine Störung des hVOR vor, dann vermindert sich der Gain-Wert des hVOR beim Videokopfimpulstest. Es treten Korrektursakkaden auf, die entweder visualisiert im Zyklus der Kopf-Augen-Bewegung verborgen sind (verdeckte, „covert" Sakkaden) oder sich danach ausbilden (sichtbare, „overt“ Sakkaden) (○ Abb. 9, siehe C Video 6 online) [41,43].

Merke: Bei peripheren Vestibulopathien können in ca. $15 \%$ verdeckte Sakkaden auftreten, die im klinischen Kopfimpulstest nicht sichtbar sind [39]. Wird der Kopf beim klinischen Kopfimpulstest um mehr als $30^{\circ}$ ausgelenkt, können verdeckte Sakkaden „demaskiert“ und damit in offene Sakkaden „umgewandelt“ werden [44]. 

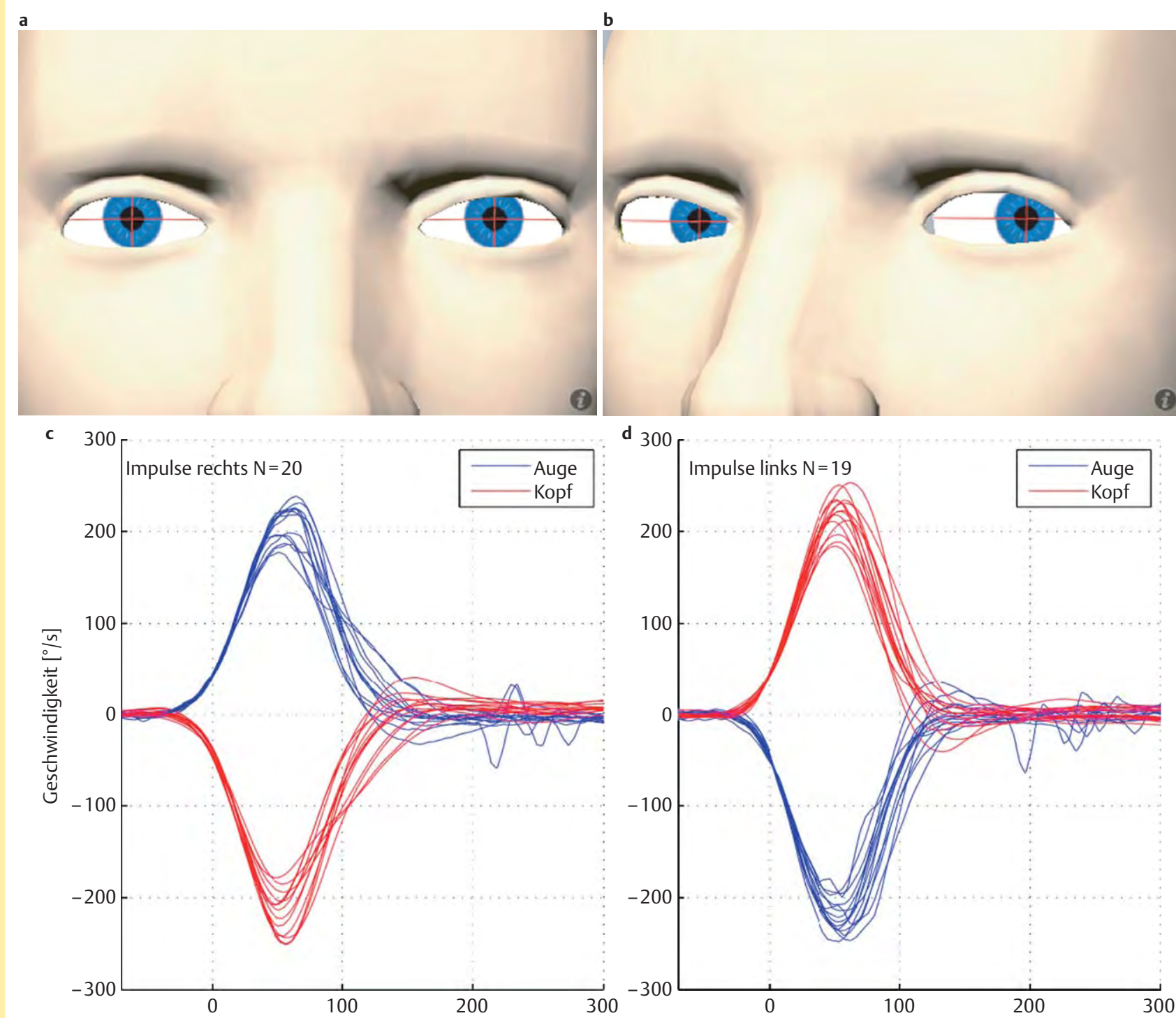

e
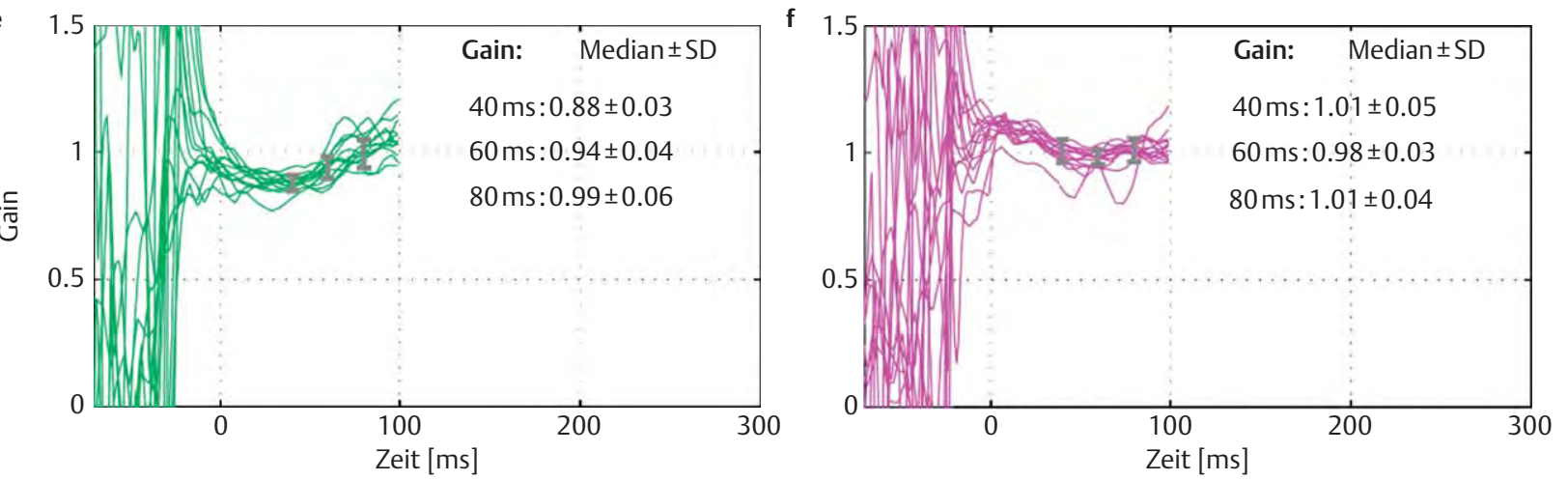

Abb. 7 Normaler klinischer und Videokopfimpulstest: a, b Screenshots der App „aVOR“; bei einem normalen vestibulookulären Reflex führt eine plötzliche, unerwartete, rasche Rotation des Patientenkopfes (ca. $15^{\circ}$ ), während der Patient ein unbewegliches Ziel fixiert, zu einer nahezu verzögerungslosen reflektorischen Augengegenbewegung ( $<10 \mathrm{~ms}=\mathrm{hVOR}$ ), sodass das Bild auf den Retinae stabilisiert bleibt. e, f Der Video-KIT objektiviert den hVOR. Das Verhältnis von Augen- und Kopfgeschwindigkeit bezeichnet man als „Gain“ und ist im vorliegenden Fall normal (siehe $\odot$ Video 5 online). 

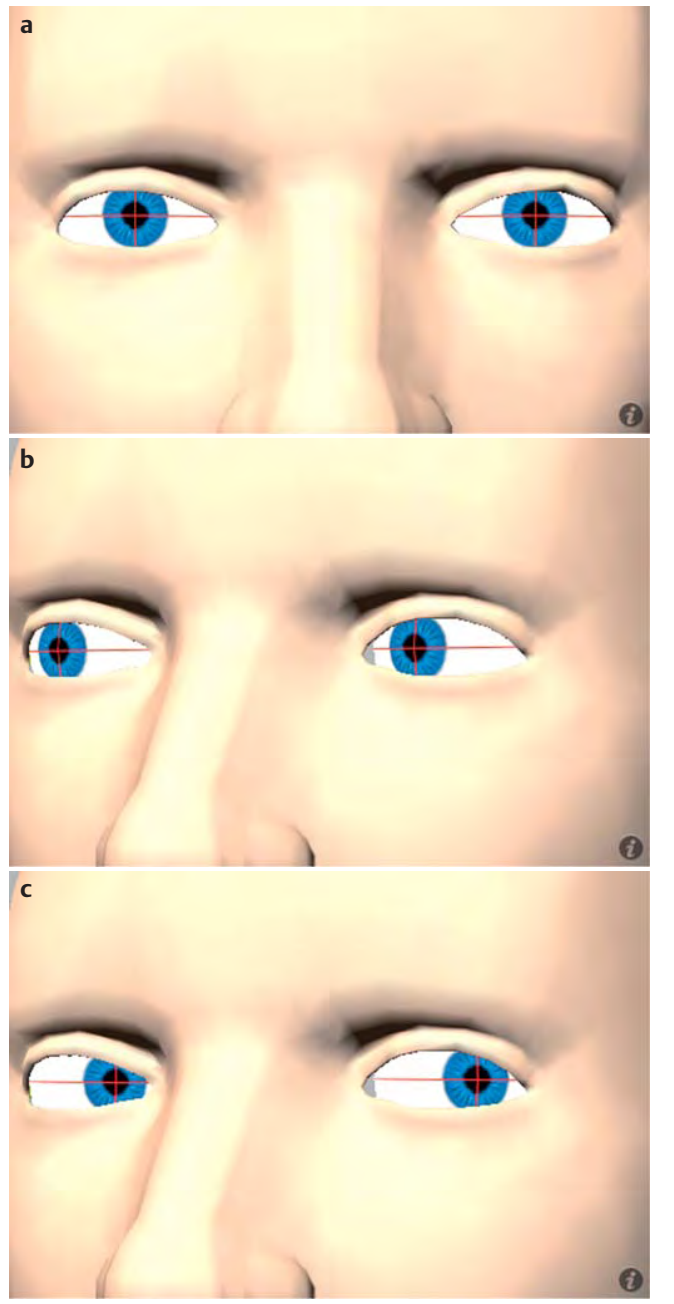

Abb. 8 a, b Sichtbare Korrektursakkaden beim klinischen Kopfimpulstest. Es liegt eine Störung des hVOR rechts vor. a Bei impulsartiger Kopfdrehung bewegen sich die Augen zunächst mit dem Kopf nach rechts mit. Danach vollziehen die Augen eine Korrekturbewegung zum fixierten stationären Blickziel („3-K-Regel“) (Screenshot aus „aVOR“ App). b Die Bickstabilisierung ist verzögert. c Eine Korrektursakkade ist sichtbar.

\section{"Schwindel" als Notfall („akutes vestibuläres Syndrom“)}

Merke: Die primäre differenzialdiagnostische Abklärung eines Patienten mit akutem Schwindel, z.B. im Rahmen der Notfallvorstellung in einer Praxis oder einer zentralen Notaufnahme einer Klinik dient zunächst der pragmatischen Frage nach einer unter Umständen bedrohlichen nichtvestibulären (z.B. kardiovaskulären) oder zentral vestibulären Ursache (z. B. Schlaganfall). Dazu gehört immer auch eine Erfassung der Vitalparameter.

Sind nichtvestibuläre Ursachen weitestgehend ausgeschlossen und keine eindeutigen „Ohrsymptome“ vorhanden, empfiehlt es sich, zunächst im Rahmen einer pragmatischen Diagnostik die folgenden Fragen zu klären:

\section{Zeigt sich im klinischen Kopfimpulstest für den horizontalen VOR (hVOR) eine Rückstellsakkade? $\nabla$}

Sind beim klinischen Kopfimpulstest reproduzierbar nachweisbare Rückstellsakkaden vorhanden (positiver klinischer KIT), so spricht dies in den meisten Fällen für ein periphere Vestibulopathie (z.B. eine akute Neuritis vestibularis). Aus dem klinischen KIT kann dann auch die aufgrund des Spontannystagmus vermutete Seitenlokalisation der Störung verifiziert werden. Ausnahmen sind z.B. laterale Ponsinfarkte, Infarkte der A. cerebellaris anterior inferior (AICA) oder Kleinhirninfarkte. Ein positiver klinischer KIT kann damit selten auch bei zentralen Störungen, z.B. bei Infarkten mit Beteiligung der vestibulären Kerngebiete auftreten. Er ist also kein ausschließliches Zeichen einer peripheren Störung. Ein negativer KIT dagegen spricht bei akutem Schwindel für ein zentrales vestibuläres Syndrom, d.h. ggf. für einen Schlaganfall (z.B. für einen Kleinhirn- oder Hirnstamminfarkt) [7,20,31-33].

\section{Liegen Augenbewegungsstörungen und Nystagmus vor?}

Zentrale Augenbewegungsstörungen finden sich häufig im Rahmen einer Akutsymptomatik assoziiert mit „Schwindel“ (akutes vestibuläres Syndrom) [28-30]. Nystagmen sind Zeichen einer vestibulären Störung. Blickrichtungsnystagmen und vertikale Spontannystagmen werden in der Regel zentral generiert und sind demnach Ursachen einer zentralen Vestibulopathie. Die Ursachen zentraler Störungen sind vielfältig und können lebensbedrohlich sein (Schlaganfall)! Aber auch chronisch-entzündliche Erkrankungen können als Erstmanifestation mit dem Primärsymptom „Schwindel“ einhergehen ( $\bullet$ Abb. 10). Handelt es sich um einen vorwiegend horizontalen oder einen horizontal rotierenden Nystagmus, muss im Hinblick auf die Differenzialdiagnose eines Schlaganfalls eine periphere Störung von einer zentralen Störung abgegrenzt werden [7,20,28-33]. Hierzu empfiehlt sich die Prüfung des VOR mittels klinischem KIT und die Klärung der Frage:

\section{Liegt eine Fehlstellung der Augenachsen (vertikale Schielstellung, „skew deviation“) vor?}

Eine vertikale Divergenz („Schielstellung“) der Augen kann durch zentrale Läsionen verursacht werden. Differenzialdiagnostisch müssen eine 


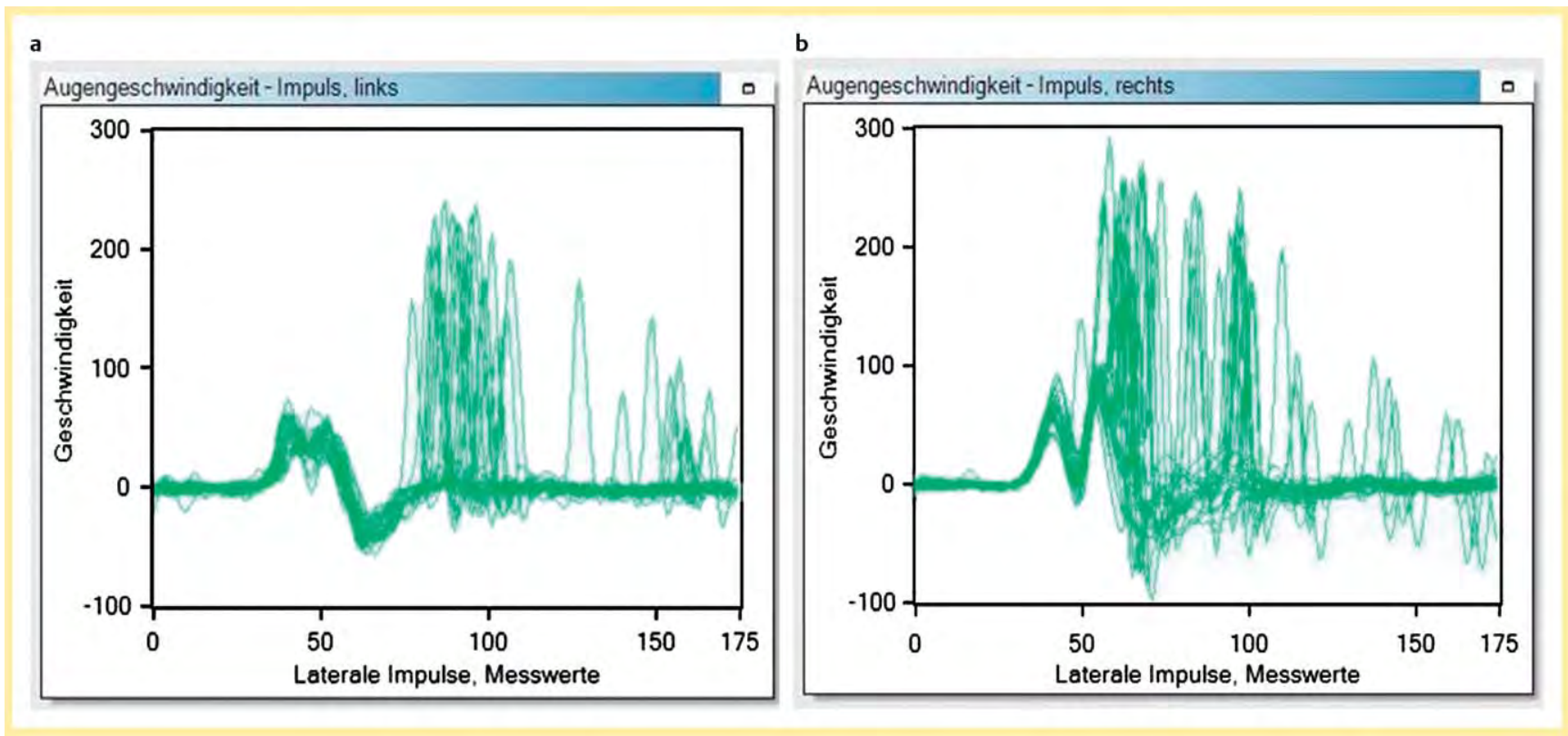

Abb. 9 a, b Videokopfimpulstest für den hVOR. a Pathologischer hVOR nach Kopfdrehung nach links mit „overt“ Sakkaden. b Pathologischer hVOR auf der rechten Seite nach Kopfdrehung nach rechts mit verdeckten „covert“ Sakkaden (im Beispiel bei einer Neuritis vestibularis rechts) (siehe $\odot$ Video 6 online).

Trochlearisparese und eine Verletzung des Auges oder eine stattgehabte Schieloperation abgegrenzt werden. Eine vertikale Divergenz wird mit dem Abdecktest diagnostiziert [7,28-30].

Kombination der 3 Screeningverfahren: "HINTS" (nach [45])

- Durchführung des klinischen Kopfimpulstests für den hVOR

- Suche nach Augenbewegungsstörungen und Nystagmus

- Suche nach Fehlstellung der Augenachsen („skew deviation“)

Cynrim et al. (2008) [46] und Newman-Toker et al. (2008) [47] haben gezeigt, dass Screeningtests, wie die Suche nach einem Defizit des hVOR (klinischer Kopfimpulstest) oder einer Fehlstellung der Augenachsen (skew deviation), eine große Bedeutung in der klinischen Notfalldiagnostik zur Differenzialdiagose des Schlaganfalls haben.

Kattah et al. führten (2009) [45] den Begriff HINTS ein (Head-Impulse-Nystagmus-Test-ofSkew). Die Kombination der 3 oben aufgeführten vestibulären Screening-Untersuchungen hat in der Frühphase einer akuten vestibulären Symptomatik (akutes vestibuläres Syndrom) eine hohe Sensitivität und Spezifität bezüglich eines Hirninfarkts, mit einer höheren Sensitivität der „HINTS“-Kombination-Tests als ein in der Frühphase durchgeführtes diffusionsgewichtetes MRT ( Tab. 6).
Merke: Mit der Kombination der 3 Screeningverfahren Kopfimpulstest, Augenbewegungsanalyse (Nystagmus) und Diagnostik einer vertikalen Divergenz („skew deviation“) „HINTS“ kann bei einer akuten Vestibulopathie mit großer Sicherheit und besser als eine MRT eine zentrale von einer peripheren Störung differenzialdiagnostisch abgegrenzt werden.

Brandt et al. (2013) [7] empfehlen ein 5-stufiges Vorgehen:

1. Test auf vertikale Schielstellung

2. Abgrenzung eines peripheren Spontannystagmus von einem zentralen Fixationsnystagmus mittel Fixationssuppression und Frenzel-Brille

3. Untersuchung eines Blickrichtungsnystagmus (zentrale Störung) entgegen der Richtung eines Spontannystagmus

4. Untersuchung auf eine Blickfolgesakkadierung (zentrale Störung)

5. Durchführung des klinischen Kopfimpulstests für den hVOR.

Man erreicht damit ebenfalls eine hohe Sensitivität und Spezifität in der Differenzialdiagnostik des Schlaganfalls.

Sprechen „HINTS“ [45] und das von Brandt et al. (2013) [7] vorgeschlagene Vorgehen nicht für eine zentrale Ursache, ist eine periphere sehr wahrscheinlich. Trotzdem kann in seltenen Fällen auch bei Vorliegen eines horizontal rotierenden Nystagmus und Fehlen aller Zeichen in der Akutphase einer zentralen Vestibulopathie (einschließlich diffusionsgewichtetem MRT) eine zentrale Ursache vorliegen. 


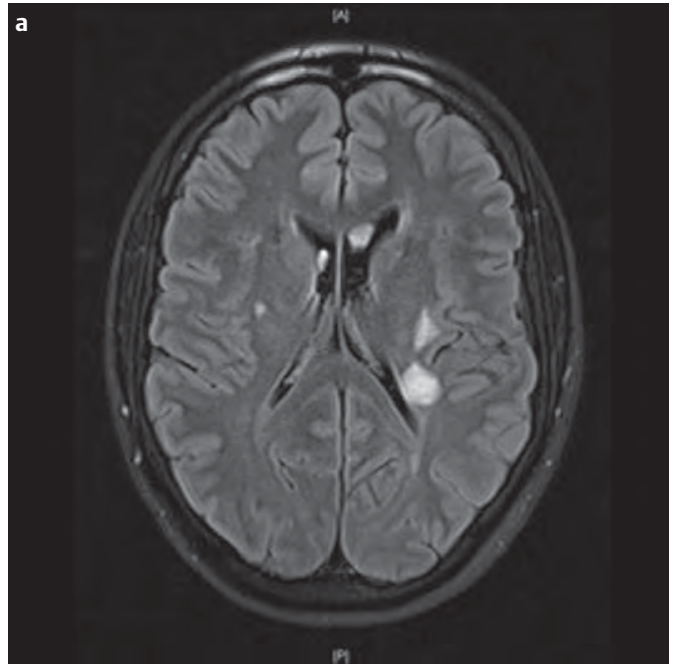

Abb. 10 a, b Fallbeispiel: Zentral vestibulärer Schwindel als Erstmanifestation einer Multiplen Sklerose: Der 20-jährige Kfz-Mechaniker stellte sich an einem Wochenende in der zentralen Notaufnahme mit Seit 2 Tagen progredientem „Schwindel“ in der zentralen Notaufnahme vor. Der Schwindel wurde überwiegend als drehend, teils als auch schwankend empfunden, begleitet von Übelkeit und Erbrechen sowie einer Fallneigung nach links. Weiterhin bestanden Doppelbilder sowie teils „milchig-trübes“ Gesichtsfeld. Hörminderung, Otalgie, Tinnitus traten nicht auf. Die Untersuchung zeigte einen horizontalen Spontannystagmus nach links mit Richtungsänderung bei Blick nach rechts (Blickrichtungsnystagmus). Der Kopfimpulstest war normal. In der MRT zeigten sich multiple, bihemisphärielle, periventri-

Findet sich eine periphere Augenbewegungsstörung, muss zur weiteren differenzialdiagnostischen Abklärung ein Augenarzt hinzugezogen werden [29,30].

Sind periphere und zentrale Ursachen eines akuten „Schwindels“ ausgeschlossen, müssen weitere differenzialdiagnostische Empfehlungen erfolgen. In unklaren Fällen empfiehlt sich dann zunächst eine Vorstellung beim Internisten, um akute kardiovaskuläre Ursachen auszuschließen. Auch psychisch bedingter „Schwindel“ kann akut auftreten. Hier ist die Anamnese zielführend (z.B. Angstsymptomatik, Panik, Depression). $\mathrm{Zu}$ berücksichtigen sind sekundäre somatoforme Schwindelformen, die häufig nach einer langwierigen peripheren Vestibulopathie (benigner paroxysmaler Lagerungsschwindel, Morbus Menière) akut auftreten können [16,17].

\section{Apparative diagnostische Verfahren und Konzepte zur Differenzialdiagnose vestibulärer Störungen}

Für die objektive Erfassung von Augenbewegungsstörungen und Nystagmus und zur Differenzialdiagnostik der funktionellen Integrität der

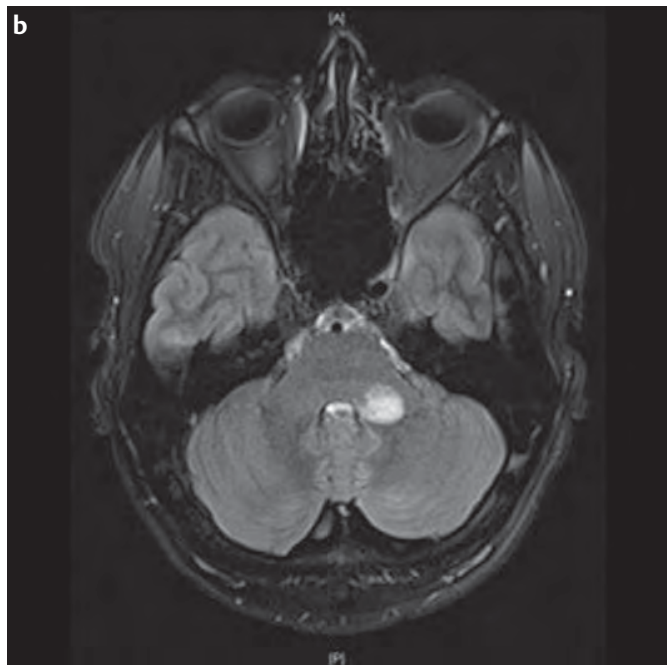

kuläre sowie tief im Marklager gelegene hyperintense, rundliche Signalanhebungen mit dem größten Befund im Pedunculus cerebelli links (13 mm), (Bild: Radiologie Universitätsklinikum Halle). Die Liquor-Punktion ergab oligoklonale Banden, autochtone IgG- und IgM-Synthese ohne Schrankenstörung und eine leichte bis mäßige Pleozytose. In neurologischen, Untersuchung fanden sich elektrophysiologisch pathologische visuelle evozierte Potenziale (VEP) und Somatosensibel evozierte Potenziale (SEP) des Nervus tibialis sowie ein erhöhter Antistreptolysintiter. Als Diagnose ergab sich eine zentrale Ursache der Beschwerden (Multiple Sklerose) als Korrelat im Rahmen einer chronisch entzündlichen Erkrankung des Zentralnervensystems einer.

5 vestibulären Rezeptoren bei peripheren Vestibulopathien stehen umfangreiche Untersuchungsverfahren zur Verfügung ( $\bullet$ Tab. 7, 8) $[7,48]$.

Merke: Vestibuläre Funktionsprüfungen prüfen die Integrität vestibulärer Reflexe, d.h. der Sinneseingänge (Rezeptoren), deren nachgeschalteter Reflexstrecke und des Endorgans. Für differenzialdiagnostische Überlegungen muss berücksichtigt werden, dass Störungen im gesamten Bereich dieser Strecke lokalisiert sein können [2].

Mit der Entwicklung neuer Tests in der Diagnostik und in Zusammenführung dieser mit bewährten Methoden ergibt sich ein Paradigmenwechsel in der Diagnostik vestibulärer Störungen [2]. Apparativ und zeitlich aufwändige Untersuchungsverfahren wurden in der letzten Zeit durch moderne, objektive, rezeptorspezifische Methoden ersetzt, z.B. zervikale und okuläre, vestibulär evozierte myogene Potenziale (cVEMP und oVEMP) und Videokopfimpulstest (vKIT). Weiterhin muss berücksichtigt werden, dass die am Orientierungssinn beteiligten Systeme eine dynamische Bandbreite besitzen, die heute durch Kombination mehrerer Testverfahren besonders für den hVOR gut erfasst werden kann [2]. 
Der vKIT und die Erfassung der dynamischen Sehschärfe (dynamic visual acuity, DVA) [50] eignen sich zur Prüfung des hochfrequenten Bereichs des hVOR bzw. der 3 Bogengänge. Thermische Prüfung und Drehprüfungen reflektieren niederfrequente Bereiche [2]. Mithilfe der vestibulär evozierten myogenen Potenziale (VEMP) können Sacculusfunktion (cVEMP, siehe Video 7 online) und überwiegende Utriculusfunktion (oVEMP) in einem breiten Frequenzband (ca. $100-4000 \mathrm{~Hz}$ ) mit unterschiedlichen Stimuli sehr variabel analysiert werden. Es ist zu berücksichtigen, dass VEMP-Untersuchungen nur die akustisch sensitiven vestibulären Typ-1-Zellen prüfen [2].

Merke: Vestibuläre Störungen weisen im zeitlichen Verlauf frequenzdynamische Besonderheiten auf, die für den hVOR besonders gut untersucht sind. Diese Kriterien können zur Differenzialdiagnostik herangezogen werden [2] (॰ Abb. 11).
Heute stehen differenzialdiagnostische Konzepte [2] zur Verfügung, mit denen eine Topodiagnostik vestibulärer Störungen unter frequenzdynamischen Aspekten erfolgen kann ( $\bullet$ Abb. 12). Nach Anamnese und orientierenden Untersuchungsverfahren erfolgt in Stufe 1 mithilfe des vKIT, der cVEMP und oVEMP ein objektives Screening der 5 Rezeptoren (Topodiagnostik) mit ersten dynamischen Aspekten.

Ergeben sich aus Stufe 1 keine Hinweise für eine Funktionsstörung, muss in Stufe 2 eine weitere frequenzspezifische Analyse der Arbeitsbereiche der geprüften Rezeptoren mit weiteren objektiven und subjektiven, dynamischen Methoden erfolgen. Hierfür eignen sich die thermische Prüfung, cVEMP und oVEMP-Untersuchungen (z.B. $250-4000 \mathrm{~Hz}$ ), die Bestimmung der subjektiven Vertikalen ggf. mit Kippung und zentrischer bzw. exzentrischer Rotation, die subjektive haptische Vertikale als Utriculus-sensitive Testmethoden und die Verifizierung der VOR-Testergebnisse mittels DVA.

Tab. 6 Englischsprachige Merkhilfe für die klinische Diagnose eines Hirnstamminfarkts.

\begin{tabular}{|l|l|l|l|}
\hline Merkwort & Englisch & Deutsch \\
\hline HINTS? & hi & head impulse test & Kopfimpulstest \\
\hline & $\mathrm{n}$ & nystagm & Nystagmus \\
\hline INFARCT! & ts & test of skew & vertikale Abweichung der Augenstellung \\
\hline & in & impulse test normal & normaler Kopfimpulstest (negativ) \\
\hline & fast phase alternating & $\begin{array}{l}\text { Richtungsänderung des Nystagmus (Blickrichtungsnys- } \\
\text { tagmus) } \\
\text { nystagm }\end{array}$ & $\begin{array}{l}\text { Refixation im Abdecktest bei vertikaler Abweichung der } \\
\text { refixation on cover test }\end{array}$ \\
\hline & &
\end{tabular}

Tab. 7 Experimentelle $\left({ }^{*}\right)$ und klinische Funktionsprüfungen für die 5 labyrinthären Rezeptoren und reflektorische Verbindungen. NF: niederfrequentes, MF: mittelfrequentes, HF: hochfrequentes Testverfahren. S: statischer Test, D: dynamischer Test. O: Objektiver Test, S: subjektiver Test., J: Ja, N: Nein,

P: Physiologischer Stimulus, U: Unphysiologischer Stimulus. SVV: Subjektive Visuelle Vertikale, SHV: Subjektive haptische Vertikale. Ungefähre Zeitangaben mit Testvorbereitung, Pausen; ** Knochenleitungsableitung und Untersuchungen bei 250, 2000-4000 Hz experimentell. *** keine eindeutige Evidenz. Nach [49].

\begin{tabular}{|c|c|c|c|c|c|c|c|c|c|}
\hline $\begin{array}{l}\text { Rezepto- } \\
\text { ren }\end{array}$ & Funktionsprüfung & $\begin{array}{l}\text { Rezeptor- } \\
\text { spezifität }\end{array}$ & $\begin{array}{l}\text { Frequenzse- } \\
\text { lektivität }\end{array}$ & $\begin{array}{l}\text { Seiten- } \\
\text { spezifität }\end{array}$ & $\begin{array}{l}\text { Statisch (S) oder } \\
\text { dynamisch (D) }\end{array}$ & $\begin{array}{l}\text { Testcha- } \\
\text { rakter }\end{array}$ & $\begin{array}{l}\text { Stimu- } \\
\text { lus }\end{array}$ & $\begin{array}{l}\text { Quantifi- } \\
\text { zierung }\end{array}$ & $\begin{array}{l}\text { Zeitauf- } \\
\text { wand (min) }\end{array}$ \\
\hline \multirow{7}{*}{$\begin{array}{l}\text { horizonta- } \\
\text { ler Bogen- } \\
\text { gang }\end{array}$} & klinischer Kopfimpulstest & ja & $\mathrm{HF}$ & ja & $\mathrm{D}$ & $\mathrm{O}$ & $\mathrm{P}$ & nein & 2 \\
\hline & Videokopfimpulstest & ja & HF & ja & D & $\mathrm{O}$ & $\mathrm{P}$ & ja & 10 \\
\hline & dynamische Sehschärfe & ja & HF & ja & $S$ & $S$ & $P$ & ja & 10 \\
\hline & thermische Prüfung Wasser & nein & NF & ja & S & $\mathrm{O}$ & U & ja & 35 \\
\hline & Drehprüfungen & nein & NF-MF & nein & D & $\mathrm{O}$ & $P$ & ja & 20 \\
\hline & Vibrationsnystagmus* & nein & HF? & ja & D & $\mathrm{O}$ & $\mathrm{P}$ & ja & 10 \\
\hline & Nahinfrarotreizung* & nein & NF & ja & $S$ & 0 & $\mathrm{P}$ & ja & 10 \\
\hline \multirow{3}{*}{$\begin{array}{l}\text { vorderer } \\
\text { Bogengang }\end{array}$} & klinischer Kopfimpulstest & ja & HF & ja & D & $\mathrm{O}$ & $\mathrm{P}$ & nein & 2 \\
\hline & Videokopfimpulstest & ja & $\mathrm{HF}$ & ja & D & $\mathrm{O}$ & $\mathrm{P}$ & ja & 10 \\
\hline & dynamische Sehschärfe & ja & $\mathrm{HF}$ & ja & D & S & $P$ & ja & 10 \\
\hline \multirow{3}{*}{$\begin{array}{l}\text { hinterer } \\
\text { Bogengang }\end{array}$} & klinischer Kopfimpulstest & ja & $\mathrm{HF}$ & ja & D & $\mathrm{O}$ & $\mathrm{P}$ & nein & 2 \\
\hline & Videokopfimpulstest & ja & $\mathrm{HF}$ & ja & D & $\mathrm{O}$ & $P$ & ja & 10 \\
\hline & dynamische Sehschärfe & ja & HF & ja & D & $S$ & $P$ & ja & 10 \\
\hline \multirow[t]{6}{*}{ Utriculus } & ACS oVEMP, BCV oVEMP** & ja & $250-4000 \mathrm{~Hz}$ & ja & $S$ & 0 & U & ja & 10 \\
\hline & SVV statisch/Kippung & ja & nein & ja & S/D & S & $\mathrm{P}$ & ja & 15 \\
\hline & SVV zentrisch/exzentrisch & ja & nein & ja & $\mathrm{D}$ & $S$ & $\mathrm{P}$ & nein & 20 \\
\hline & SHV & nein & nein & ja & D & S & $\mathrm{P}$ & ja & 15 \\
\hline & exzentrische Rotation & ja & $\mathrm{NF}-\mathrm{MF}$ & ja & D & 0 & $\mathrm{P}$ & ja & 20 \\
\hline & Head Heave Test* & nein & $\mathrm{HF}$ & ja & D & $\mathrm{O}$ & $\mathrm{P}$ & ja & 10 \\
\hline \multirow[t]{2}{*}{ Sacculus } & AC cVEMP, BCV cVEMP** & ja & $250-4000 \mathrm{~Hz}$ & ja & $S$ & $\mathrm{O}$ & U & nein & 10 \\
\hline & Schaumstoff-Posturografie ${ }^{* * *}$ & nein & NF & nein & D & S & $\mathrm{P}$ & ja & 10 \\
\hline \multirow{2}{*}{$\begin{array}{l}\text { Otolithen- } \\
\text { organe }\end{array}$} & langsamer Tandem-Gang & nein & NF & nein & D & $S$ & $\mathrm{P}$ & nein & 2 \\
\hline & Schrägachsenrotation & nein & NF-MF & nein & $\mathrm{D}$ & 0 & $\mathrm{P}$ & ja & 25 \\
\hline
\end{tabular}




\begin{tabular}{|c|c|c|}
\hline $\begin{array}{l}\text { Thermische } \\
\text { Prüfung }\end{array}$ & $\begin{array}{c}\text { Rotatorische } \\
\text { Tests }\end{array}$ & $\begin{array}{l}\text { Kopfimpuls- } \\
\text { test }\end{array}$ \\
\hline niederfrequente & mittelfrequente & hochfrequente Testverfahren \\
\hline
\end{tabular}

Abb. 11 Arbeitsbereiche des hVOR und Erfassung mit diagnostischen Methoden. Modifiziert nach [2].

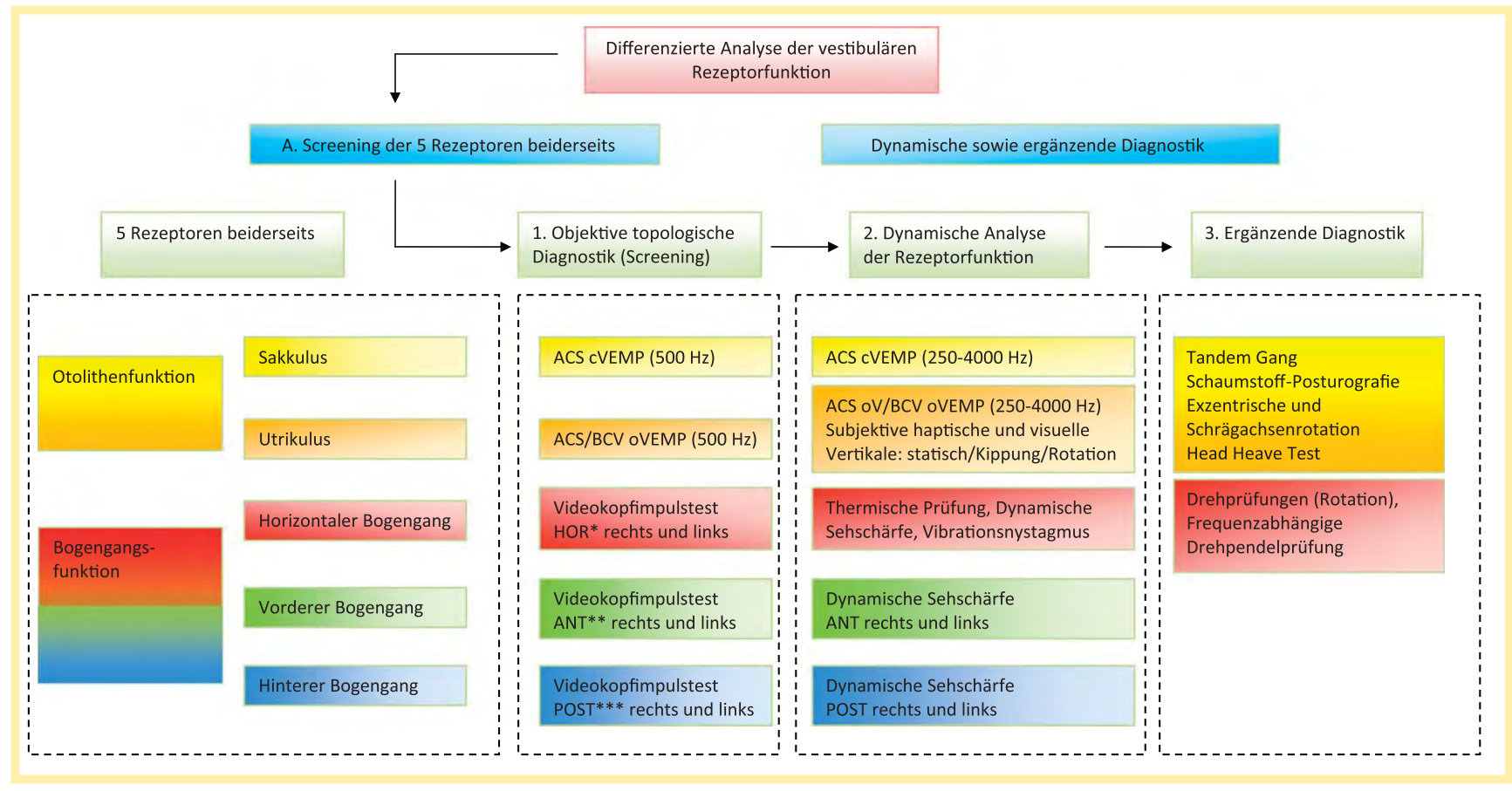

Abb. 12 Differenzierte klinische Rezeptorfunktionsanalyse. ${ }^{*}$ HOR horizontaler, ${ }^{* *}$ ANT vorderer, ${ }^{* * *}$ POST hinterer Bogengang. ACS: Luftleitung, BCV: Knochenleitung. Nach [2].

Ergeben sich aus diesen Untersuchungen keine eindeutigen Hinweise auf eine rezeptorbedingte Störung unter dynamischen Aspekten, oder sind die Untersuchungen nicht einsetzbar (z. B. Schallleitungsstörung, Affektionen des äußeren und Mittelohrs, angeborener Pendelnystagmus) müssen in Stufe 3 ergänzend weitere objektive, subjektive und unspezifische z.T. aufwendige Verfahren eingesetzt werden (z.B. Drehprüfungen), um einer Diagnose nahezukommen bzw. diese zu bestätigen [2].

Tab. 8 Ungefähre Schwellen für den Nachweis eines peripheren vestibulären Spontannystagmus mit unterschiedlichen Erfassungsmethoden.

\begin{tabular}{|l|l|}
\hline Erfassungsmethode & $\begin{array}{l}\text { Ungefähre Erken- } \\
\text { nungsschwelle [ } \% \text { s] }\end{array}$ \\
\hline visuell & $>4$ \\
\hline Frenzel-Brille & $>0,5$ \\
\hline Elektronystagmografie & $<0,5$ \\
\hline Videonystagmografie & $<0,5$ \\
\hline Modifiziert nach [7] &
\end{tabular}

\section{Differenzialdiagnostische Aspekte wichtiger Krankheitsbilder}

\section{Benigner paroxysmaler Lagerungsschwindel (BPPV) \\ $\nabla$}

Besteht der Verdacht auf einen benignen paroxysmalen Lagerungsschwindel, müssen definierte Lagerungsmanöver zur weiteren differenzialdiagnostischen Abgrenzung und Lokalisation des betroffenen Bogengangs erfolgen. Je nachdem, ob sich das Partikelkonglomerat beim BPPV im Endolymphsystem (Kanalolithiasis) oder auf der Kupula (Kupulolithiasis) befindet, findet sich ein spezifischer $\mathrm{VOR}<1 \mathrm{~min}$ (siehe Video 8, 9 und 10 online). Bei der Kupulolithiasis und bei der Kanalolithiasis der lateralen Bogengänge kann die Dauer auch länger sein $[7,8,36,51,52]$.

Man unterscheidet derzeit 3 Formen des BPPV:

- degenerativ bedingter BPPV

- idiopathischer BPPV

- symptomatischer BPPV [36,51].

Der degenerative BPPV kommt im höheren Lebensalter vor, da die Wahrscheinlichkeit der Ablösung von Partikeln aus dem Bereich der utriku- 
Tab. 9 Unterschiede des degenerativen, idiopathischen und symptomatischen (posttraumatischen) BPPV Nach [36].

\begin{tabular}{|c|c|c|c|c|c|c|}
\hline BPPV & Ursache & $\begin{array}{l}\text { Bevorzugte Lokali- } \\
\text { sation }\end{array}$ & Seitenprävalenz & $\begin{array}{l}\text { Alters- und Ge- } \\
\text { schlechtsprävalenz }\end{array}$ & Rezidive & Therapie \\
\hline $\begin{array}{l}\text { degene- } \\
\text { rativ }\end{array}$ & $\begin{array}{l}\text { degenerative Verände- } \\
\text { rungen, wahrschein- } \\
\text { lich Partikel aus dem } \\
\text { Utriculus }\end{array}$ & $\begin{array}{l}\text { monokanalikulär (hin- } \\
\text { terer Bogengang), in } \\
\text { anderen Bogengängen } \\
\text { seltener }\end{array}$ & selten bilateral & $\begin{array}{l}\text { Frauen häufiger, } \\
\text { meist im höheren } \\
\text { Lebensalter }\end{array}$ & seltener & $\begin{array}{l}\text { einfacher, } \\
\text { Spontanremis- } \\
\text { sion häufiger }\end{array}$ \\
\hline $\begin{array}{l}\text { idiopa- } \\
\text { thisch }\end{array}$ & ungeklärt & $\begin{array}{l}\text { vorwiegend monoka- } \\
\text { nalikulär (hinterer und } \\
\text { lateraler Bogengang) }\end{array}$ & selten mehrere Bogengänge & keine & seltener & $\begin{array}{l}\text { einfacher, } \\
\text { Spontanremis- } \\
\text { sion häufiger }\end{array}$ \\
\hline $\begin{array}{l}\text { symptoma- } \\
\text { tisch (z. B. } \\
\text { posttrau- } \\
\text { matische) }\end{array}$ & $\begin{array}{l}\text { Absprengung von Oto- } \\
\text { konien durch Kopfan- } \\
\text { prall, vermutlich mehr } \\
\text { intakte Otokonien }\end{array}$ & $\begin{array}{l}\text { monokanalikulär (hin- } \\
\text { terer Bogengang), in } \\
\text { anderen Bogengängen } \\
\text { häufiger }\end{array}$ & $\begin{array}{l}\text { keine Seitenprävalenz, } \\
\text { häufig bilateral }\end{array}$ & $\begin{array}{l}\text { jedes Geschlecht, } \\
\text { jedes Alter }\end{array}$ & häufiger & schwieriger \\
\hline
\end{tabular}
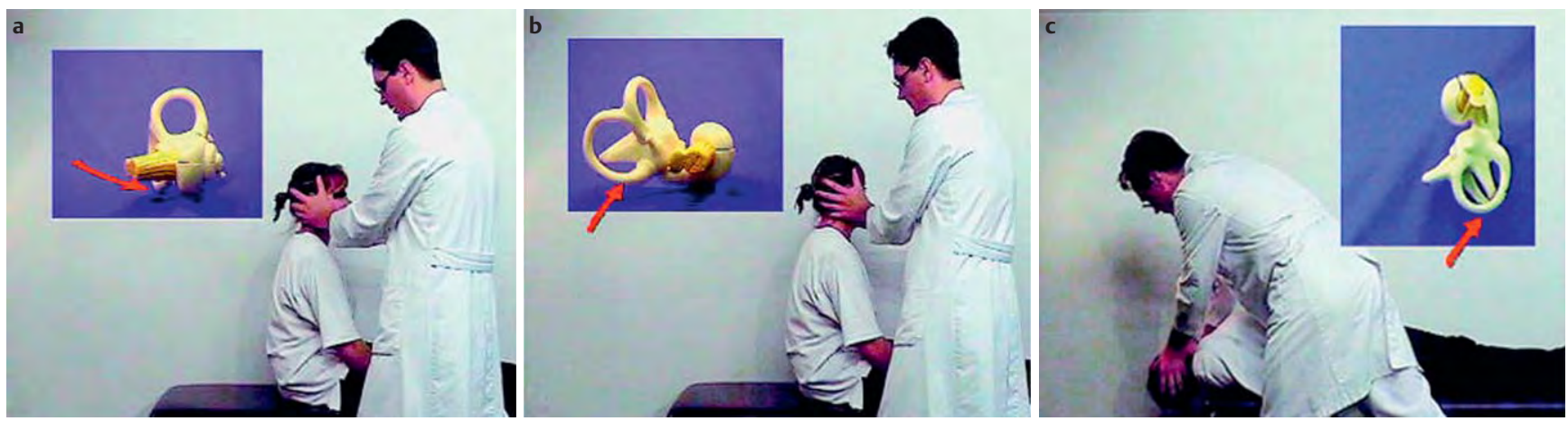

Abb. 13 Dix-Hallpike-Manöver (bei Verdacht auf benignen paroxysmalen Lagerungsschwindel des linken hinteren Bogengangs). Der rote Pfeil zeigt auf die Lage des Partikelkonglomerats im Endolymphsystem (Kanalolithiasis). Charakteristisch für eine Kanalolithiasis des posterioren Bogengangs ist ein mit einigen Sekunden Latenz auftretender Crescendo-Decrescendo- (stärker werdender, dann abnehmender) Schwindel von bis zu 60s Dauer, der von einem rotatorischen Nystagmus zum unten liegenden, betroffenen Ohr begleitet ist. Beim Aufrichten kehrt sich die Nystagmusrichtung um. Bei Lagerungsmanöver in Richtung der nicht erkrankten Seite tritt kein „Schwindel“ auf (Seitenbestimmung). Aus: Walther LE. Wiederherstellende Verfahren bei gestörtem Gleichgewicht Laryngo-Rhino-Otol 2005; 84: 70-91 [52].

\section{Erratum}

Die Quelle für die Bilder

in Abb. 13 auf S. 560

wurde angegeben mit

„Walther LE.

Wiederherstellende Verfahren bei gestörtem Gleichgewicht LaryngoRhino-Otol 2005; 84: 70 -91“. Die korrekte Quellenangabe muss für diese und auch in der als Quelle angegebenen Publikation lauten: „Bilder aus Lehrvideo $\odot$ Prof. Dr. med. Martin Westhofen und Dipl. Ing. Marita Dreckmeyer M. A., 2002 (Bildrechte bei den Autoren); Universitätsklinik für Hals-Nasen-

Ohrenheilkunde und Plastische Kopf- und Halschirurgie Aachen.“ lären Otokonienmembran aufgrund morphologischer Veränderungen der Otokonien im Alter zunimmt. So beträgt die Häufigkeit eines BPPV im 8. Dezenium bis zu $10 \%$.

Der idiopathische BPPV betrifft vorwiegend jüngere Altersgruppen und ist mit der Hypothese einer Degeneration daher nicht ohne Weiteres zu erklären. Er verhält sich jedoch ähnlich.

Symptomatische Formen kommen z.B. im Rahmen einer „Neuritis“ vestibularis, eines Morbus Menière, aber auch nach Kopfanpralltraumen und Explosionstraumen vor. Liegt ursachlich ein Trauma vor, dann spricht man vom posttraumatischen BPPV. Die posttraumatische Variante des symptomatischen BPPV weist einige Besonderheiten auf. So wird beschrieben, dass die Rezidivrate posttraumatischer Fälle nach einer Behandlung höher sein soll ( $\bullet$ Tab. 9) [36,50-52].

Merke: Beim benignen paroxysmalen Lagerungsschwindels sind die hinteren (posterioren) Bogengänge (ca. 75\%) häufiger als die horizontalen (lateralen Bogengänge) (ca. 20\%) und die vorderen (superioren) Bogengänge (ca. 5\%) betroffen.
Cave: Ein BPPV tritt meistens im hinteren Bogengang und häufiger rechts auf. Bei der Differenzialdiagnostik sollte das diagnostische Lagerungsmanöver zuerst für den rechten hinteren Bogengang durchgeführt werden (cave: Abschwächung der Reaktion bei Reizwiederholung!).

Beim BPPV zeigen die Lagerungsmanöver für die jeweiligen Bogengänge eine spezifische Nystagmusreaktion (VOR) ( Abb. 13, 14). Die Richtung des Nystagmus entspricht einer Augendrehachse, die senkrecht zur Ebene des erregten Bogengangs liegt. Neben diesem Merkmal sind die kurze Latenz (5-15s), die kurze Dauer ( $<1 \mathrm{~min}$, bei der Kupulolithiasis auch länger) und die Ermüdbarkeit die klassischen Zeichen ( $\bullet$ Tab. 10) $[7,8,51,52]$.

Merke: Die Latenz des Nystagmus beim hBPPV ( $h=$ horizontaler Bogengang) ist länger als beim pBPPV ( $\mathrm{p}=$ posteriorer Bogengang) und aBPPV ( $a$ =anteriorer Bogengang). Bei zentralen Störungen findet sich keine oder nur eine sehr kurze Latenz (1-5s). 


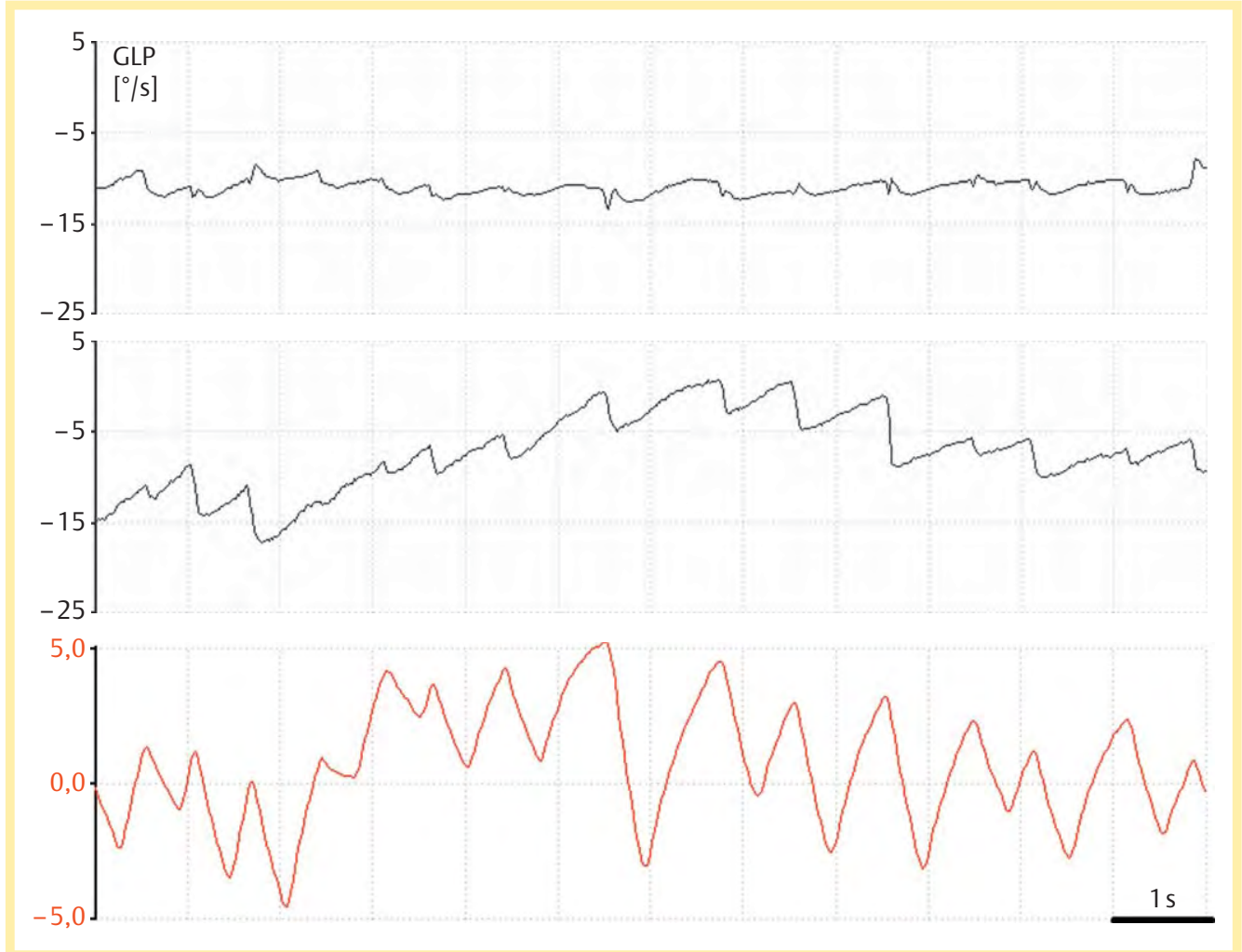

Abb. 14 Video-okulografische Dokumentation eines BPLS des hinteren Bogengangs rechts ausgelöst durch das diagnostische DixHallpike-Lagerungsmanöver. Dominierende torsionale Komponente des induzierten vestibulookulären Reflexes (rot), sehr geringe horizontale (oben) und stärkere vertikale Augenbewegung (Mitte), Geschwindigkeit der langsamen Nystagmusphase (GLP) [\% $\%$. Aus [36].

Tab. 10 Lagerungsmanöver zur Differenzierung eines benignen paroxysmalen Lagerungsschwindels der posterioren (p), horizontalen (h) und anterioren (a) Form des BPPV (Kanalolithiasis).

\begin{tabular}{|c|c|c|}
\hline Bogengänge & Lagerungsmanöver & Nystagmusreaktion (VOR) \\
\hline $\begin{array}{l}\text { hintere Bogengän- } \\
\text { ge (pBPPV) }\end{array}$ & $\begin{array}{l}\text { Dix-Hallpike-Manöver (siehe } \bigcirc \text { Video } 11 \\
\text { online; [53]) }\end{array}$ & $\begin{array}{l}\text { torsionell-linear mit Upbeat-Komponente } \\
\text { (geotrop: in das erkrankte, unten liegende } \\
\text { Ohr), Umkehr bei Wiederaufrichten }\end{array}$ \\
\hline $\begin{array}{l}\text { horizontale Bo- } \\
\text { gengänge (hBPPV) }\end{array}$ & $\begin{array}{l}\text { „Supine Roll Test“: in Rückenlage: schnelle } \\
\text { Seitwärtsdrehung des Kopfes um } 90^{\circ} \text { oder kom- } \\
\text { plette Kopf-Körper-Drehung (siehe } \\
\text { online; [53]) Video } 12\end{array}$ & $\begin{array}{l}\text { horizontal, stärker in das betroffene (unten } \\
\text { liegende [geotrop] Ohr) }\end{array}$ \\
\hline $\begin{array}{l}\text { vordere Bogen- } \\
\text { gänge (aBPPV) }\end{array}$ & Dix-Hallpike-Manöver (s. o.) & $\begin{array}{l}\text { geotrop, linear-torsionell mit Downbeat- } \\
\text { Komponente (in das erkrankte, oben } \\
\text { liegende Ohr), Umkehr bei Wiederaufrichten }\end{array}$ \\
\hline
\end{tabular}

Cave: Ein BPPV kann in seltenen Fällen zentralvestibulär bedingt sein (z. B. Tumore der hinteren Schädelgrube, $\bullet$ Abb. 15). Bei Therapieresistenz muss eine Bildgebung veranlasst werden.

\section{Morbus Menière}

Der Morbus Menière ist eine rezidivierende idiopathische Erkrankung des Innenohrs, die mit einem Endolymphhydrops assoziiert ist und mit Drehschwindelanfällen, Attacken von Schallempfindungsschwerhörigkeit sowie mit Tinnitus und Druck- oder Völlegefühl einhergeht.

Grundlage für die differenzialdiagnostische Einordnung ist die Definition der American Academy of Otolaryngology \& Head and Neck Surgery aus dem Jahre 1995 [11], die wiederkehrende Drehschwindelattacken von $>20$ min in Kombination mit einer (audiometrisch dokumentier-

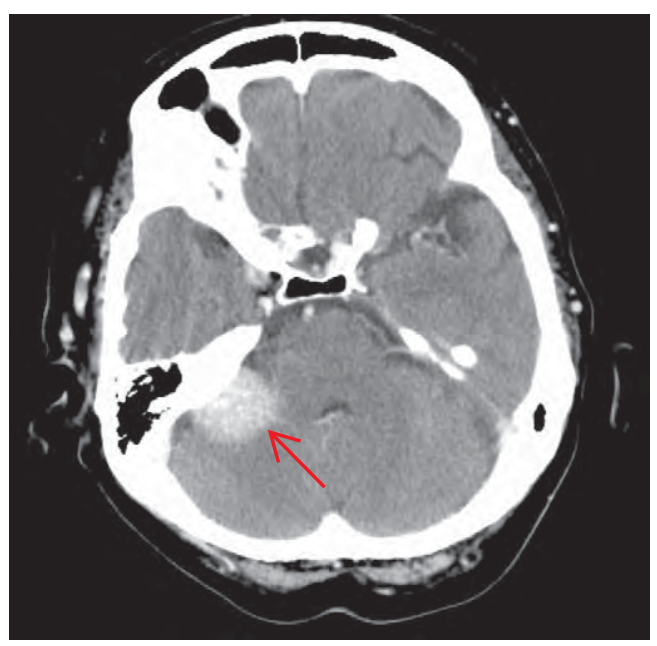

Abb. 15 Seltene Differenzialdiagose eines benignen paroxysmalem Lagerungsschwindels: Meningeom in der hinteren Schädelgrube. 
ten), in der Regel fluktuierenden Hörminderung im Tieftonbereich, fluktuierendem Tinnitus sowie Ohrdruck voraussetzt.

Eine mögliche Erkrankung mit einem Schwindelereignis ohne nachweisbare Defizite wird von einem wahrscheinlichen (mindestens 1 Schwindelereignis und dokumentierter Hörverlust) und einem eindeutigen („definite“) Morbus Menière (mindestens 2 Schwindelereignisse und dokumentierter Hörverlust) unterschieden.

Ein gesicherter Morbus Menière kann nur auf der Basis des Nachweises eines Endolymphhydrops gestellt werden [11].

Im Frühstadium der Erkrankung bilden sich alle Symptome nach einem Schwindelanfall zurück, sodass sich funktionsdiagnostisch keine Defizite der Hörfunktion sowie der Rezeptorfunktion finden (möglicher Morbus Menière). Die Diagnosestellung in diesem Krankheitsstadium stützt sich allein auf die Anamnese. Daher müssen weitere Differenzialdiagnosen abgegrenzt werden.

Ein Sekundenschwindel spricht mehr für eine vaskuläre Genese. Auch Lage- oder Lagerungsabhängigkeiten spielen bei der Abgrenzung eine wesentliche Rolle: Eine orthostatische Dysregulation geht neben Drehschwindel auch mit einem „Schwarzwerden vor Augen“ einher und dauert nur wenige Sekunden.

Bei einem BPPV dauert eine Schwindelepisode meistens nicht länger als $1 \mathrm{~min}$. Zumeist ereignet sich ein gutartiger Lagerungsschwindel typischerweise z. B. beim Drehen im Bett oder beim Bücken und Wiederaufrichten. Tinnitus und eine Hörstörung treten hierbei nicht auf, Probleme können aber entstehen, wenn diese bereits einseitig vorbestehen oder ein therapieresistenter oder rezidivierend auftretender Lagerungsschwindel vorliegt.

Bei der vestibulären Migräne kann die typische Trias des Morbus Menière ebenfalls temporär auftreten. Eine Abgrenzung ist in vielen Fällen nicht einfach. Der MR-morphologische Nachweis eines Endolymphhydrops kann in solchen Fällen Klarheit schaffen [54]. Wenn die Abgrenzung nicht gelingt, ist eine probatorische Behandlung mit Betablockern, Valproinsäure oder Topiramat möglich.

Bakterielle oder virale Infektionen mit Innenohrbeteiligung (Otitis media, „Grippeotitis“) lassen sich mit dem Leitsymptom Ohrenschmerzen abgrenzen.

Seltener sind akute, nur Sekunden bis wenige Minuten dauernde Schwindelepisoden, bei denen es zu einseitigem Tinnitus und/oder einer Hörminderung während der Attacken oder im Verlauf kommen kann, wenn diese durch eine neurovaskuläre Kompression aufgrund des Kontakts des N. vestibulocochlearis mit einer Gefäßschlinge, z.B. der A. cerebelli anterior inferior, hervorgerufen werden $[7,55,56]$.
Merke: Isolierte akute Hörstörungen im niedrigen Frequenzbereich („Tieftonhörsturz“/Fluktuierendes Tieftongehör) stellen per definitionem auch in Kombination mit Tinnitus (noch) keinen Morbus Menière dar, gelten aber als ein Risikofaktor für das spätere Auftreten des Vollbildes eines M. Menière. Begriffe, wie „atypischer“ oder insbesondere „monosymptomatischer Morbus Menière" sollten nicht verwendet werden. D.h. die aktuell gültige Klassifikation der AAO-HNS als derzeit weltweit akzeptierter Konsens sollte einheitlich angewandt werden.

Es ist heute allgemein akzeptiert, dass der endolymphatische Hydrops, also eine Zunahme der Endolymphflüssigkeit, das pathophysiologische Korrelat bei einem Morbus Menière darstellt [57], auch wenn nicht jede Homöostasestörung des Innenohres mit einem endolymphatischen Hydrops zur Ausbildung des klinischen Bildes eines Morbus Meniere nach Definition der AAOHNS führt [58]. Um die Diagnose zu stützen, können der Nachweis eines Hydrops mittels Elektrokochleografie [59] und ein Dehydratationstest nach Klockhoff und Lindblom 1966 [60] erfolgen. Neuere Untersuchungen zeigen die Möglichkeit der Darstellung eines endolymphatischen Hydrops mittels MRT nach Gadoliniumgabe auf [61-63].

Treten beim Patienten mit einem Morbus Menière Stürze mit erhaltenem Bewusstsein auf („drop attacks“) muss eine Störung der Otolithenfunktion (Sacculus) angenommen werden. In solchen Fällen ist die Ableitung der VEMP sinnvoll [7,24,52].

\section{Neuritis vestibularis superior und inferior \\ $\nabla$}

Die Neuritis vestibularis zählt neben dem benignen paroxysmalen Lagerungsschwindel und dem Morbus Menière zu den häufigsten peripheren Vestibulopathien. Die Symptomatik ist definiert durch prolongierten Drehschwindel, das Vorliegen eines horizontal rotierenden Spontannystagmus, Fallneigung und Gangabweichung zur erkrankten Seite und vegetative Symptome (Übelkeit und Erbrechen) [7,64-66].

Diagnostisch zeigen sich eine pathologische visuelle subjektive Vertikale (in der Akutphase), ein pathologischer KIT sowie ein pathologisches Seitenverhältnis bei der thermischen Prüfung. Eine serologische Untersuchung mit dem Hintergrund einer vermuteten Reaktivierung von Herpesviren ist diagnostisch nicht zielführend und therapeutisch nicht relevant [67]. Bei einer akuten Neuritis vestibularis lassen sich heute mithilfe der aktuellen Funktionsprüfungen, insbesondere mit- 
hilfe des KIT, der cVEMP (Sacculus) und oVEMP (überwiegender Utriculusanteil) 3 Konditionen differenzieren $[68,69]$ ( $\odot$ Abb. 16):

1. Schädigung der Pars superior (,akute Neuritis des N. vestibularis superior"), d.h. der Rezeptoren des horizontalen und vorderen Bogengangs sowie des Utriculus und der neuralen Strukturen, deren Afferenzen in den N. vestibularis superior münden.

2. Affektion der Pars inferior, d. h. der Rezeptoren und Afferenzen des hinteren Bogengangs und des Sacculus (,akute Neuritis des N. vestibularis inferior“), welche zum N. vestibularis verlaufen $[68,69]$. Für die Neuritis des N. vestibularis inferior mit Affektion des hinteren Bogengangs und Funktionsstörung des Sacculus treffen die Diagnosekriterien der „Neuritis vestibularis“ nicht zu. Dabei liegt meistens sowohl eine normale thermische Erregbarkeit als auch ein regelrechter KIT bei der Prüfung des horizontalen VOR beiderseits vor. Der KIT des oberen (vorderen) Bogengangs ist regelrecht, der KIT des hinteren Bogengangs der erkrankten Seite ist häufig pathologisch $[68,69]$.

3. Vollständiger peripher-vestibulärer „Ausfall“ mit Störung von Pars superior und Pars inferior („akute Neuritis vestibularis superior et inferior").

Bei allen 3 Subklassifikationen lassen sich partielle oder vollständige Funktionsstörungen nachweisen. Am häufigsten ist die Schädigung der Pars superior.

Merke: Im Rahmen der „Erholung“ der Funktion einer Neuritis vestibularis zeigt das hochfrequente Frequenzspektrum des hVOR meist noch Beeinträchtigungen, während der niederfrequente Bereich (thermische Prüfung) bereits normal ist. Beim Morbus Menière ist die Frequenzbeeinträchtigung invers $[2,64,65,70,71]$. Bei der Neuritis vestibularis regeneriert sich die Otolithenfunktion im Fall einer „Erholung“ eher als der hVOR [71].

Die differenzialdiagnostische Abgrenzung zu einem Morbus Menière kann Probleme bereiten, wenn sich dieser im Rahmen eines episodischen, lang andauernden Anfalls (z.B. bis zu 24h) erstmalig manifestiert. Eine klare Differenzierung kann in der Akutphase nicht vorgenommen werden.

Auch eine zentrale vestibuläre Störung muss in die differenzialdiagnostischen Überlegungen einbezogen werden [72]. Die Durchführung von „HINTS“ ist daher obligat. Das Wechseln der Nystagmusrichtung im Zeitverlauf kann ein Hinweis für einen Morbus Menière sein.

\section{Dehiszenzsyndrome \\ $\nabla$}

Das von Minor et al. [13] vor 15 Jahren beschriebene Dehiszenzsyndrom des vorderen (oberen) Bogengangs ist ein seltenes, aber differenzialdiagnostisch wichtiges Krankheitsbild. Es zeichnet sich durch eine Vielzahl vestibulärer und auditorischer Symptome aus, die Anlass zu einer weiteren differenzierten Funktionsdiagnostik sein müssen. Wichtige differenzialdiagnostische Überschneidungen bestehen zu einer Vestibularisparoxysmie, aber auch zu einem Morbus Menière und zu einer Otosklerose.

Merke: Ein Dehiszenzsyndrom muss differenzialdiagnostisch von einem Morbus Menière und einer Otosklerose abgegrenzt werden.

Knöcherne Dehiszenzen können sich im Bereich der Konturen aller 3 Bogengänge befinden. Meistens ( $>80 \%$ ) ist der vordere, selten sind der hintere und laterale Bogengang ( je $<10 \%$ ) betroffen [12-14,73].

Im Rahmen der differenzialdiagnostischen $\mathrm{Ab}$ klärung empfiehlt sich folgende Vorgehensweise [73]:

- Der Stimmgabeltest nach Weber lateralisiert beim einseitigen Vorliegen einer Dehiszenz meist in das betroffene Ohr (Schallleitungsstörung im Tieftonbereich).

- Bei der Durchführung des „Malleolus-Tests“, bei dem die angeschlagene Stimmgabel auf den Knöchel des Patienten gesetzt wird, wird die fernab über das Knochengerüst eingegebene Schallenergie eventuell im erkrankten Ohr verstärkt wahrgenommen.

- Im Tonaudiogramm kann eine Schallleitungsschwerhörigkeit vorliegen, die der Konstellation der Knochen-Luftleitungs-Kurve einer Otosklerose (wichtige Differenzialdiagnose vor allem vor Durchführung einer Stapedotomie) ähneln kann. Die Maxima der Schallleitungskomponente finden sich bei $250-500 \mathrm{~Hz}$.

- Bei der Durchführung der okulären, vestibulär evozierten myogenen Potenziale (oVEMP) in Luftleitung zeigen sich in vielen Fällen verminderte Reizschwellen (!) und mehrfach erhöhte Amplitudenkomplexe auf dem erkrankten Ohr im Vergleich zu einer Normalpopulation bzw. im Vergleich zum Gegenohr, wenn dieses nicht betroffen ist. Da okuläre VEMPs im Vergleich zu Gesunden beim Dehiszenzsyndrom auch noch im Hochfrequenzbereich nachweisbar sind, sollte eine VEMP-Multifrequenzanalyse (z.B. 100-4000 Hz) in Luft- und ggf. Knochenleitung erfolgen.

- Zu berücksichtigen ist, dass die betroffenen Patienten unter einem positiven Tullio-Phänomen leiden können. Aus diesem Grund ist eine simultane Elektro- oder videonystagmografische Aufzeichnung bei den VEMP-Untersu- 
Abb. 16 a-d Neuritis vestibularis rechts. Die 48-jährige Patientin stellte sich in der zentralen Notaufnahme mit plötzlich aufgetretenem, andauerndem Drehschwindel, Übelkeit und Erbrechen vor. Hörminderung, Otalgie und Tinnitus wurden verneint. Die klinische Untersuchung zeigte einen horizontalen Spontannystagmus nach links, eine Gangabweichung und Fallneigung nach rechts. Der klinische Kopfimpulstest zeigte Rückstellsakkaden bei horizontaler Kopfbewegung nach rechts (siehe Video 13). Mit dem Videokopfimpulstest (hVOR) a,b konnten overt-Sakkaden rechts objektiviert werden. e Der Gain rechts war vermindert, $\mathbf{c}, \mathbf{d}$ links normale Abfolge der Kopfbewegungen. Die am Folgetag durchgeführte thermische Prüfung zeigte eine fehlende Erregbarkeit rechts. f Normales Audiogramm beiderseits.
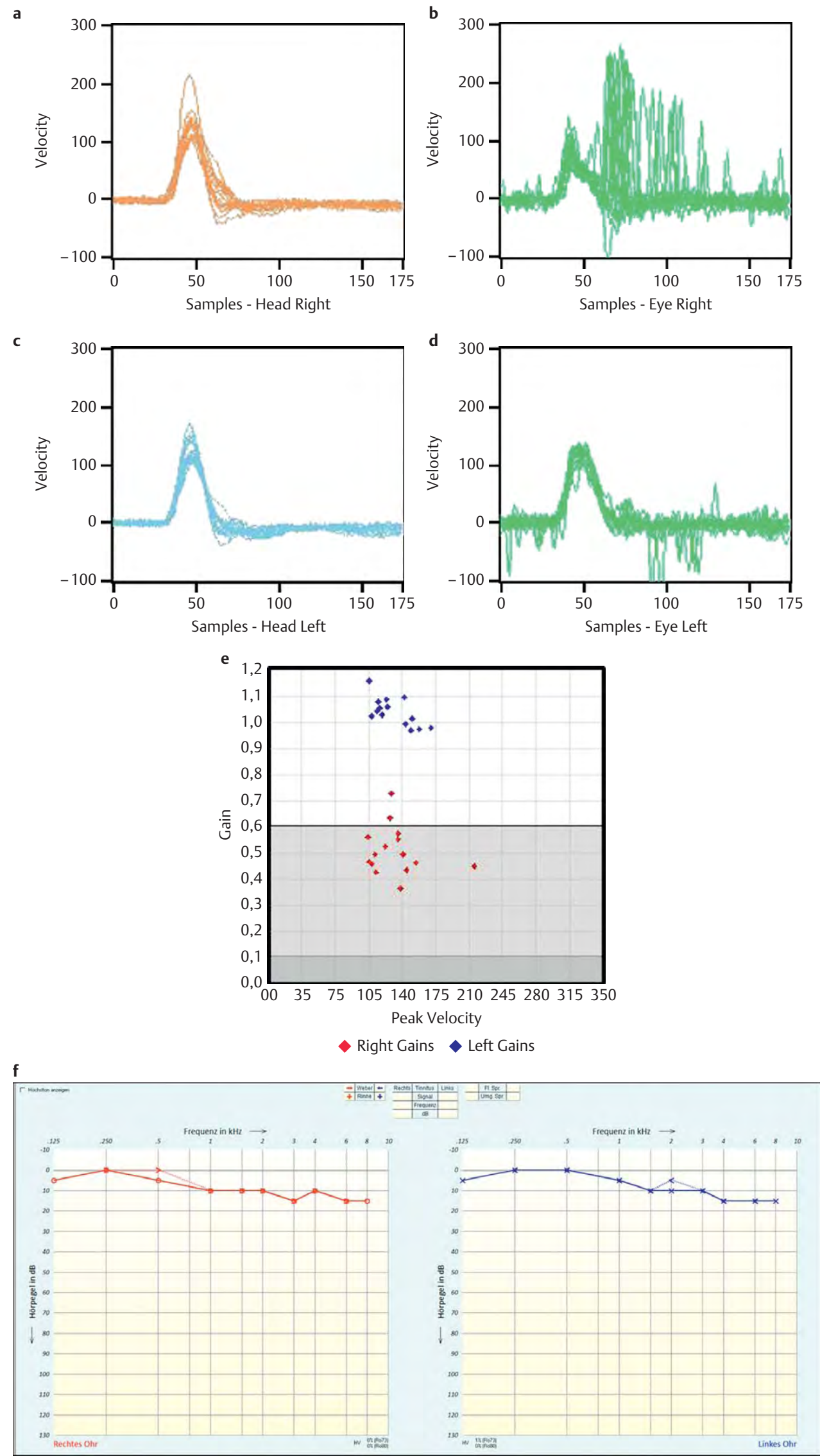
chungen zu empfehlen. Druckinduzierter „Schwindel“ kann durch Prüfung des Tulliound Hennebert-Zeichens mit den entsprechenden Provokationsmanövern getestet werden. Gleichzeitig empfiehlt sich auch hier eine Dokumentation eines eventuell induzierten Nystagmus. Außerdem beklagen viele Patienten eine Autophonie bzw. die verstärkte Wahrnehmung körpereigener Geräusche.

- Der klinische und der Videokopfimpulstest zeigen in der Ebene des betroffenen Bogengangs ggf. eine pathologische Rückstellsakkade. Mithilfe des Videokopfimpulstests kann der „Gain“ in der Ebene des betroffenen Bogengangs (RALP/LARP) sowohl objektiviert als auch quantifiziert werden.

- Die Elektrokochleografie ist ebenfalls ein sinnvolles Element in der Diagnosefindung einer Dehiszenz und zeigt bei Patienten mit einer Dehiszenz ein vergrößertes Verhältnis $(>0,4)$ von Summationspotenzial zu Aktionspotenzial (SP-AP-Ratio), ähnlich wie bei Patienten mit kochleärem Hydrops beim Morbus Menière. Beim Verdacht auf ein Dehiszenzsyndrom kann sie daher als weiterer diagnostischer Baustein eingesetzt werden.

- Erhärtet sich der Verdacht auf ein Dehiszenzsyndrom, muss eine computertomografische Untersuchung veranlasst werden. Um die Dehiszenz zu erfassen, ist eine Rücksprache mit dem Radiologen bezüglich der Verdachtsdiagnose, der Schnittführung und der erforderlichen Schichtdicke der Aufnahme erforderlich. So ist eine hochauflösende CT der Otobasis im Bereich des Labyrinths mit Rekonstruktion in der Ebene des superioren Bogengangs und orthogonal zu dieser erforderlich. Ausschlaggebend für die Diagnose einer Dehiszenz sollte eine Kombination aus klinischen, radiologischen und diagnostischen Tests sein. Fehlen vestibuläre Symptome sowie eine Hörstörung bei einem computertomografischen Nachweis einer Dehiszenz, liegt kein klinisch relevantes, behandlungsbedürftiges Krankheitsbild vor ( $\bullet$ Abb. 17).

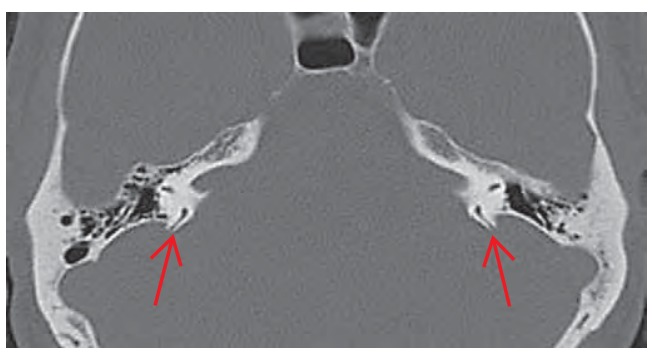

Abb. 17 Dehiszenzsyndrom. Computertomografische Darstellung (axial): fehlende knöcherne Bedeckung der beiden hinteren Bogengänge („drittes Fenster“; rote Pfeile).
Merke: Beweisend für ein Dehiszenzsyndrom ist die Kombination aus klinischer Symptomatik, funktionsdiagnostischen Befunden und dem Nachweis einer Dehiszenz in der hochauflösenden computertomografischen Untersuchung der Otobasis.

\section{Richtungsweisende diagnostische}

Befunde beim Dehiszenzsyndrom

- Schwindel mit Nystagmus (torsionelle Augenbewegungen) bei intensiver akustischer Reizung (Tullio-Phänomen)

- ipsiversive Kopfbewegungen bei akustischer Reizung

- Nystagmus beim Valsalva-Versuch (Verifizierung mittels Video- oder Elektronystagmografie)

- Nachweis einer Schallleitungsstörung im niederfrequenten Bereich

- verminderte Schwellen (!) und erhöhte Amplituden bei VEMP-Untersuchungen

- computertomografischer Nachweis einer Dehiszenz oder einer nur sehr dünnen Knochenbedeckung („Beinahe-Dehiszenz“)

Beim kürzlich beschriebenen Krankheitsbild der „Beinahe-Dehiszenz“ finden sich ähnliche klinische und diagnostische Befunde. Allerdings ist die Knochenschale über dem betroffenen Bogengang sehr dünn, aber nicht dehiszent $[73,74]$.

\section{Bilaterale Vestibulopathie}

Das klassische Bild einer bilateralen Vestibulopathie ist gekennzeichnet durch eine Funktionsstörung des hVOR auf beiden Seiten. Häufige Ursachen sind eine ototoxische Medikation (Aminoglykoside), ein bilateraler Morbus Menière sowie eine Meningitis.

Bei der Beeinträchtigung der Blickstabilisierung können keine Ersatzsysteme einspringen, somit ergeben sich bei schnellen Kopfbewegungen Probleme, sie sich als Oszillopsien äußern. Zudem finden sich eine Stand- und Gangunsicherheit im Dunkeln sowie eine Störung des Raumgedächtnisses und der Navigation.

Patienten mit einer länger bestehenden bilateralen Vestibulopathie und geringen Beschwerden zeigen nach unseren bisherigen Untersuchungen mittels Video-KIT (hVOR) vorwiegend verdeckte Rückstellsakkaden. Je nach Affektion des Hochfrequenzbereichs des VOR bzw. Beteiligung der anderen Bogengänge zeigt der KIT eine Beeinträchtigung. Die thermische Erregbarkeit ist beidseits vermindert oder nicht nachweisbar $[7,52]$. 

risschwannom links. Die Patientin zeigte ein fast normales Hörvermögen. Der Videokopfimulstest zeigte einzelne covert Sakkaden, der Gain war vermindert, die thermische Prüfung war pathologisch ( $>25 \%$ Seitendifferenz). Zervikale und okuläre VEMP waren regelrecht.
Abb. 18 a, b Vestibula-
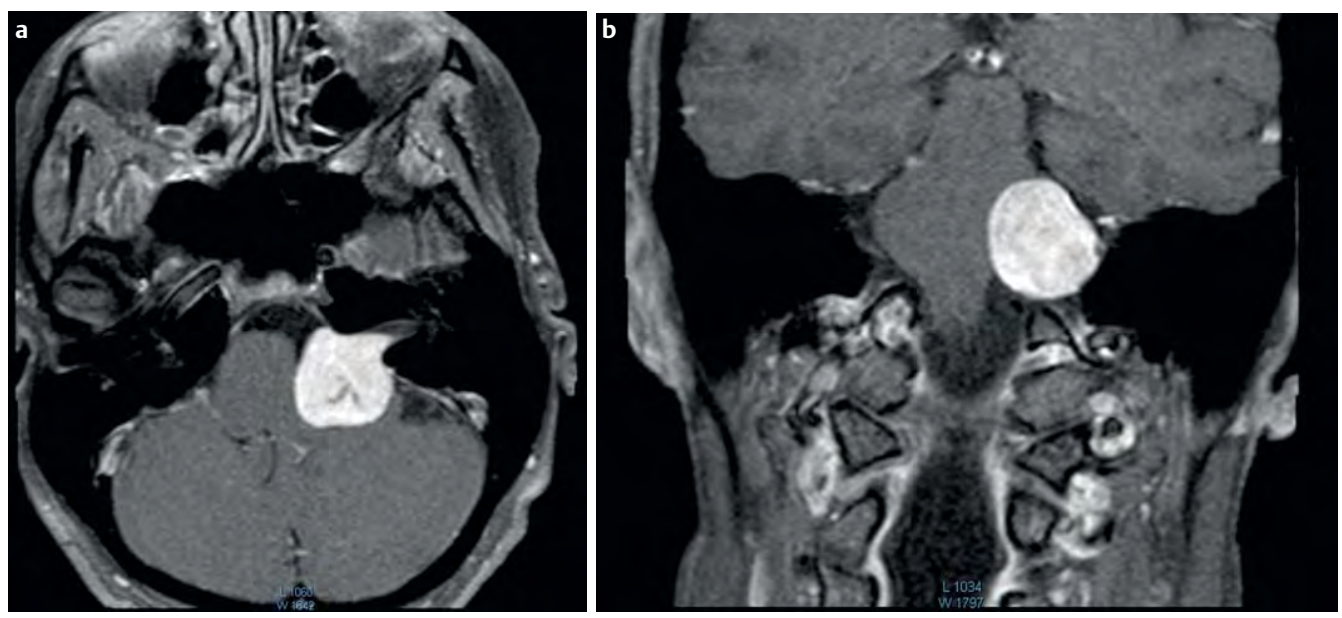

Abb. 19 Intravestibuläres Schwannom (MRT axial). Der Pfeil zeigt die Auslöschung des Flüssigkeitssignales im rechten Vestibulum. Durch das langsame Wachstum verursachte die Pathologie relativ geringe und nur unspezifische Schwindelbeschwerden aber einen akuten Hörverlust (Bild: Neuroradiologie Univ. Tübingen).

den, da diese häufig nur bei hoher Auflösung in der Bildgebung und besonderer Beachtung der labyrinthären Strukturen entdeckt werden (๑ Abb. 19).

Zur Einschätzung von Schwindelbeschwerden, insbesondere zur Beurteilung der Funktion des horizontalen vestibulookulären Reflexes (hVOR), war die thermische Prüfung (Kalorik) bisher der Standardtest. Neuere Testverfahren, wie für die Bogengänge der Videokopfimpulstest, bereichern die Schwindeldiagnostik und ermöglichen eine quantitative, seiten- und rezeptorspezifische

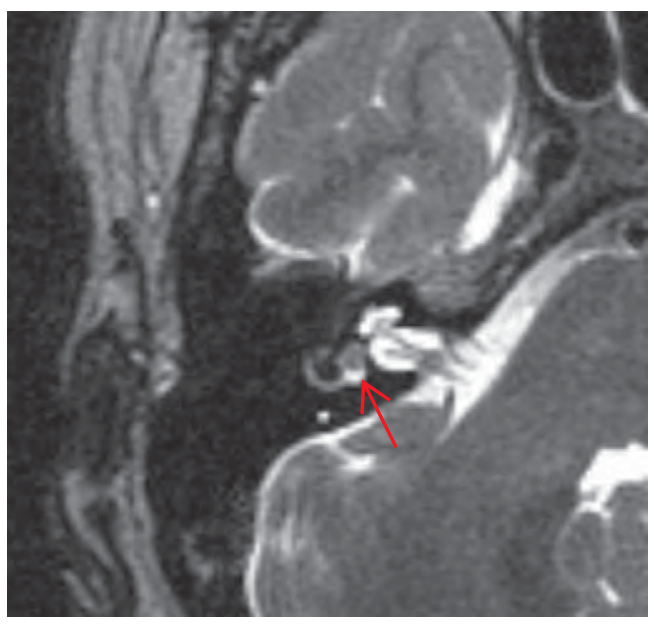
Funktionsprüfung der 5 vestibulären Endorgane bzw. deren reflektorischer Verbindungen $[38,76]$.

Merke: Die thermische Prüfung ist beim Vestibularisschwannom in den meisten Fällen pathologisch, während der hochfrequente Bereich des hVOR (VKIT) noch normale Testergebnisse aufweisen kann [38].

Vestibularisschwannome (oft auch noch als „Akustikusneurinom“ bezeichnet) gehen von den Schwann-Zellen des N. vestibulocochlearis aus, die Pars vestibularis superior (ca. 20\%) und der N. cochlearis (1\%) sind seltener betroffen als die Pars vestibularis inferior (ca. 80\%) [38,75]. Das progressive Tumorwachstum führt beim VS zu Hörverlust (85-95\%), Tinnitus (56-70\%) und Schwindel mit Unsicherheitsgefühl und Balancedefiziten (46-70\%).

Die Einteilung der Tumorstadien wird heute entsprechend der Hannover-Klassifikation T1-T4 (intra-/extrameataler Tumor ohne/mit Hirnstammkompression) vorgenommen [75].

Die Symptomatik ist jedoch aufgrund des langsamen Wachstums nicht eindeutig ( $\bullet$ Abb. 18). Primärsymptom kann eine akute Hörstörung sein, die sich als „Hörsturz“ präsentiert. Akute vestibuläre Symptome sind hingegen selten, sodass es im Verlauf der Größenzunahme des Tumors zu einer vestibulären Kompensation kommen kann und dem Symptom „Schwindel“ eher als Begleitsymptom eine untergeordnete Rolle zukommt. Auch an die Möglichkeit eines intravestibulären Schwannoms sollte gedacht wer-

\section{Zoster oticus \\ $\nabla$}

Beim Zoster oticus kommt das Leitsymptom „Schwindel“ in ca. 75\% der Fälle in Kombination mit herpetiformen Effloreszenzen im Ohrbereich vor ( $\bullet$ Abb. 20). Eine frühe Diagnosestellung wird kompliziert durch uncharakteristische, subjektive Beschwerden („Schwindel“, Ohren- und Kopfschmerzen) in der präeruptiven (Prodromal-) Phase. Die differenzialdiagnostische Abgrenzung kann dann Probleme bereiten.

Eine anfängliche Rötung des Gehörgangs kann als Otitis externa imponieren. Effloreszenzen im Gehörgangs- und Trommelfellbereich mit hämorrhagischer Begleitkomponente stehen der Otitis externa haemorrhagica et bullosa („Grippe-Otitis“) nahe. Bei „Schwindel“ sollte mit den zur Verfügung stehen Mitteln (Frenzel-Brille, Video- oder Elektronystagmografie) nach Störungen des VOR gefahndet werden. 
Die akute Symptomatik entspricht der einer akuten Neuritis vestibularis. Gleichzeitig können andere Hirnnerven (N. facialis, kochleärer Anteil des N. vestibulocochlearis) betroffen sein. Sehr selten fehlen diese begleitenden Hirnnervenaffektionen. In solchen Fällen ist die Abgrenzung zu einem Herpes simplex schwierig.

Ein Eczema herpeticatum, ein bullöses Erysipel und autoimmun-bullöse Dermatosen kommen vor allem dann in Frage, wenn simultan ohrferne Dermatome betroffen sind. Hilfreich sind eine serologische Diagnostik, VZV-IgA-, IgM- und IfGAntikörper-Bestimmungen. Ein positives VZVIgA ist ein zuverlässigerer Indikator für eine

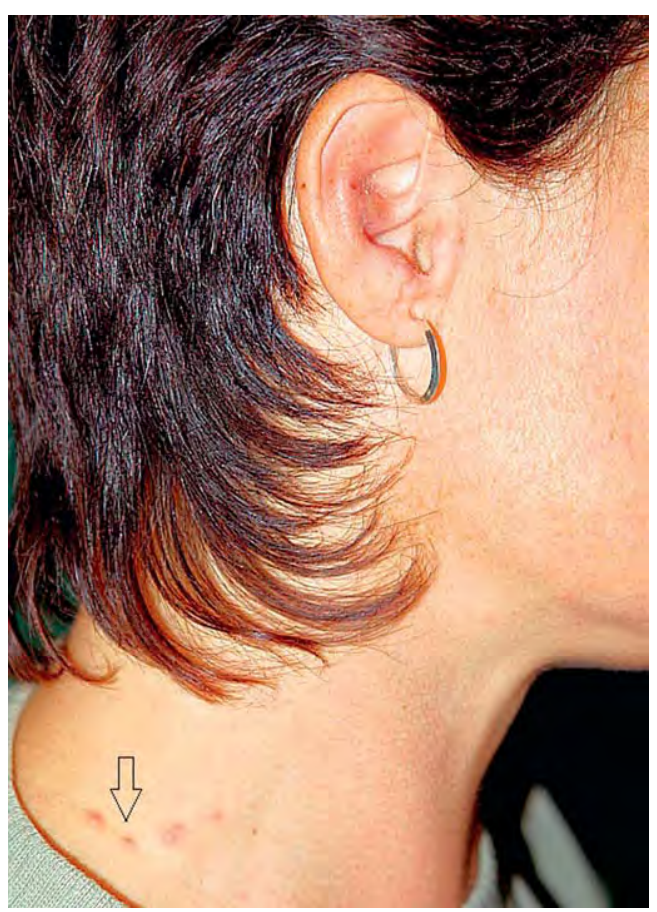

Abb. 20 Zoster oticus: Simultanes Vorkommen hämorrhagischer Effloreszenzen im Cavum conchae und in einem zervikalen Dermatom rechts (selten). Aus [77].
Zoster-Infektion als ein positives ZV-IgM. Die Serokonversion zu IgA- und IgM-Antikörpern dauert bis zu 1 Woche nach dem Auftreten der ersten Effloreszenzen [77].

\section{Weitere otologische Ursachen von Schwindel}

$\nabla$

Zu den für den Hals-Nasen-Ohrenarzt aufgrund der charakteristischen Anamnese bzw. Begleitsymptome einfach zu diagnostizierenden otologischen Schwindelursachen gehören die Labyrinthfistel bei Cholesteatom und traumatische Labyrinthausfälle bei Schädel-Hirn-Traumata mit Schläfenbeinfraktur ( $\bullet$ Abb. 21).

\section{Danksagung}

Wir danken Herr PD Dr. rer. nat. rer. medic. habil. T. Rahne und Herrn I. Seiwerth für die Unterstützung bei den Fallbeispielen und bei der Videoerstellung.

\section{Literatur}

1 Straumann D, Müri RM, Hess K. Neurootologie und Neuroopthalmologie. In: Hess K, Steck AJ, Hrsg. Kompendium der Neurologie. Göttingen: Hans Huber, 2002; 355-372

2 Walther LE. Application of the new diagnostic tests for vertigo. Differentiated analysis of vestibular function. HNO 2013; 61: 730-737

3 Bisdorff A, Von Brevern M, Lempert T, Newman-Toker $D$. Classification of vestibular symptoms: towards an international classification of vestibular disorders. J Vestib Res 2009; 19: 1-13

4 Bisdorff A, Bosser G, Gueguen R, Perrin P. The epidemiology of vertigo, dizziness, and unsteadiness and its links to co-morbidities. Front Neurol 2013; 22: 29

5 Bisdorff A. Schwindel und Kopfschmerz. Psychiatrie \& Neurologie 2013; 1: 16-18

6 Walther LE. Diagnostik und Therapie vestibulärer Störungen. In: Biesinger A, Iro H, Hrsg. Schwindel. Berlin: Springer, 2007; 60-78

7 Brandt T, Dieterich M, Strupp M. Vertigo-Leitsymptom Schwindel. Berlin: Springer, 2013; 1-142
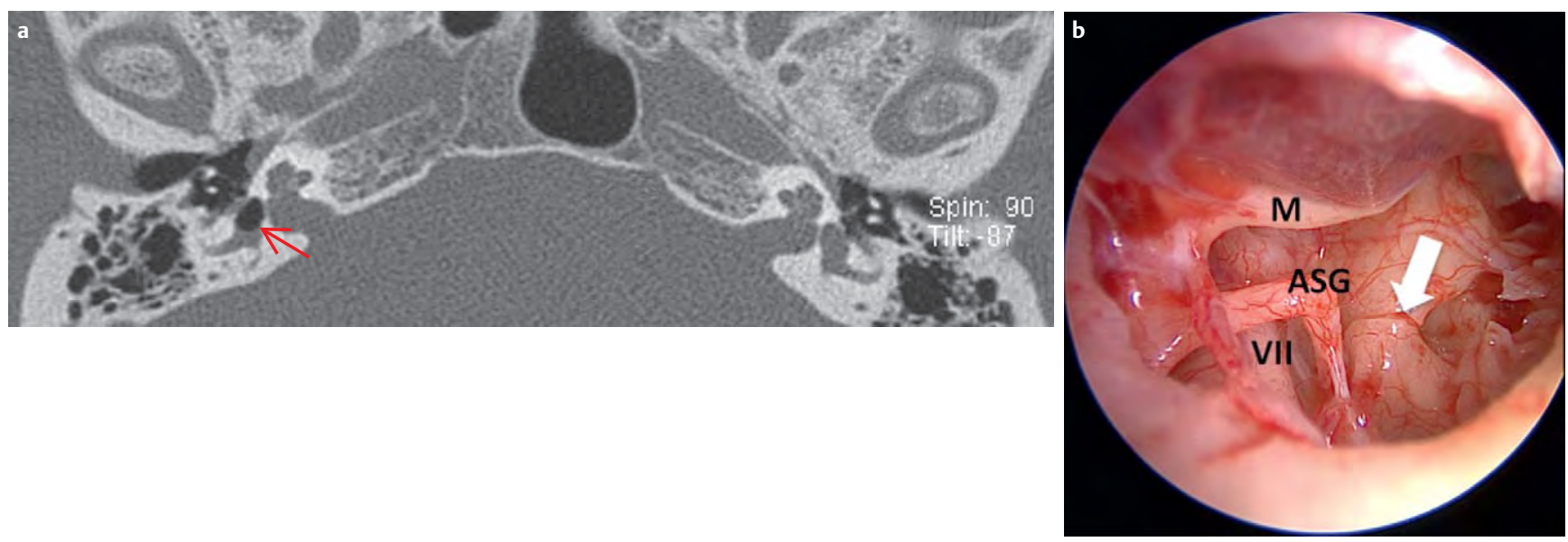

Abb. 21 a, b Schläfenbeinfraktur mit Pneumolabyrinth. a Im CT (nativ, axial) zeigt sich ein Lufteinschluss im rechten Vestibulum (Pfeil). b Intraoperativ zeigt sich der Frakturspalt (Pfeil) durch die basale Kochleawindung in die ovale Fensternische. M: Hammergriff; ASG: Amboss-Steigbügel-Gelenk; VII: N. facialis. (CT: Radiologie Martin-Luther Universität Halle/Saale). Neben einem Spontannystagmus zeigten sich im Intervall im vKIT „overt“ Sakkaden sowie eine fehlende thermische Erregbarkeit rechts. 
8 Brandt T, Steddin S. Current view of the mechanism of benign paroxysmal vertigo: cupulolithiasis or canalolithiasis? J Vest Res 1993; 3: 373-382

9 Steddin S, Brandt T. Benign paroxysmal positional vertigo. Differential diagnosis of posterior, horizontal and anterior canalolithiasis. Nervenarzt 1994; 65: 505-510

10 Lempert T, Olesen J, Furman J et al. Vestibular migraine: diagnostic criteria. J Vest Res 2012; 22: 167-172

11 American Academy of Otolaryngology-Head and Neck Foundation. Committee on Hearing and Equilibrium guidelines for the diagnosis and evaluation of therapy in Menière's disease. Am Acad Otolaryng Head Neck 1995; 113: 181-185

12 Minor LB. Clinical manifestations of superior semicircular canal dehiscence. Laryngoscope 2005; 115 : 1717-1727

13 Minor LB 1, Solomon D, Zinreich JS, Zee DS. Soundand/or pressure-induced vertigo due to bone dehiscence of the superior semicircular canal. Arch Otolaryngol Head Neck Surg 1998; 124: 249-258

14 Luers JC, Hüttenbrink KB. Acoustic and vestibular effects of superior semicircular canal dehiscence. HNO 2013; 61: 743-749

15 Ernst A, Basta D. Gleichgewichtsstörungen. Stuttgart: Thieme, 2012

16 Eckhardt-Henn A, Tschan R, Best C, Dieterich M. Somatoform vertigo syndrome. Nervenarzt 2009; 80: 909-917

17 Eckhardt-Henn A. Psychosomatic vertigo disorders. HNO 2013; 61: 777-780

18 Staab JP, Ruckenstein MJ. Chronic dizziness and anxiety: effect of course of illness on treatment outcome. Arch Otolaryngol Head Neck Surg 2005; 131: 675-679

19 Langhagen $T$, Lehnen $N$, Krause E, Jahn K. Vertigo in children and adolescents. Part 1: Epidemiology and diagnosis of peripheral vestibular disorders. HNO 2013; 61: 791-802 quiz 803-804

20 Walther LE, Hülse $R$, Blödow $A$. Dizziness from the viewpoint of otorhinolaryngology. Ophthalmologe 2013; 110: 16-25

21 Walther LE, Kleeberg J, Rejmanowski G et al. Falls and fall risk factors. Are they relevant in ENT outpatient medical care? HNO 2012; 60: 448-456

22 Walther LE, Nikolaus T, Schaaf H, Hörmann K. Vertigo and falls in the elderly. Part 1: epidemiology, pathophysiology, vestibular diagnostics and risk of falling. HNO 2008; 56: 833-841 quiz 842

23 Walther LE, Nikolaus T, Schaaf H, Hörmann K. Vertigo and falls in the elderly: Part 2: Fall diagnostics, prophylaxis and therapy. HNO 2008; 56: 927-936 quiz 937

24 Calzada AP, Lopez IA, Ishiyama G, Ishiyama A. Otolithic membrane damage in patients with endolymphatic hydrops and drop attacks. Otol Neurotol 2012; 33: 1593-1598

25 Volz-Sidiropoulou E, Takahama J, Gauggel S, Westhofen $M$. „Dizziness Handicap Inventory“: Erste psychometrische Kennwerte einer Deutschen Version. Laryngo-Rhino-Otol 2010; 89: 418-423

26 Gloor-Juzi T, Kurre A, Straumann D, de Bruin ED. Translation and validation of the vertigo symptom scale into German: A cultural adaption to a wider German-speaking population. BMC Ear Nose Throat Disord 2012; 12: 7

27 Kurre A, van Gool CJ, Bastiaenen $\mathrm{CH}$ et al. Translation, cross-cultural adaptation and reliability of the german version of the dizziness handicap inventory. Otol Neurotol 2009; 30: 359-367

28 Thömke F. Augenbewegungsstörungen. Stuttgart: Thieme, 2008

29 Strupp M, Walther LE, Eckhardt-Henn A, Franko Zeitz $P$. Diagnosis of vertigo: keep an eye on central eye movement disorders. Ophthalmologe 2013; 110: 31-38

30 Strupp M, Hüfner K, Sandmann $R$ et al. Central Oculomotor Disturbances and Nystagmus: A Window into the Brainstem and Cerebellum. Dtsch Arztebl Int 2011; 108: 197-204
31 Ahn BY, Bae JW, Kim DH et al. Pseudovestibular neuritis associated with isolated insular stroke. J Neurol 2010; 257: 1570-1572

32 Kremmyda O, Kirchner H, Glasauer S et al. False-positive head-impulse test in cerebellar ataxia. Front Neurol 2012; 3: 162

33 Lee H, Sohn SI, Cho YW et al. Cerebellar infarction presenting isolated vertigo - frequency and vascular topographical patterns. Neurology 2006; 67: 1178-1183

34 Choi KD, Kim JS. Head-shaking nystagmus in central vestibulopathies. Ann N Y Acad Sci 2009; 1164: 338-343

35 Strupp M, Brandt T. Peripheral vestibular disorders. Curr Opin Neurol 2013; 26: 81-89

36 Walther LE, Brusis T. Of the expert office: expert evaluation of benign paroxysmal positional vertigo (BPPV) to current criteria. Laryngo-Rhino-Otol 2012; 91: 36-39

37 Halmagyi GM, Curthoys IS. A clinical sign of canal paresis. Arch Neurol 1988; 45: 737-739

38 Blödow A, Blödow J, Bloching MB et al. Horizontal VOR function shows frequency dynamics in vestibular schwannoma. Eur Arch Otorhinolaryngol 2014 [Epub ahead of print]

39 Blödow A, Pannasch S, Walther LE. Detection of isolated covert saccades with the video head impulse test in peripheral vestibular disorders. Auris Nasus Larynx 2013; 40: 348-351

40 Ulmer E, Chays A. Curthoys and Halmagyi Head Impulse test: an analytical device. Ann Otolaryngol Chir Cervicofac 2005; 122: 84-90 [in French]

41 Weber KP, MacDougall HG, Halmagyi GM, Curthoys IS. Impulsive testing of semicircular-canal function using video-oculography. Ann N Y Acad Sci 2009; 1164: 486-491

42 Bartl K, Lehnen N, Kohlbecher S, Schneider E. Head impulse testing using video-oculography. Ann N Y Acad Sci 2009; 1164: 331-333

43 MacDougall HG, McGarvie LA, Halmagyi GM et al. Application of the video head impulse test to detect vertical semicircular dysfunction. Otol Neurotol 2013; 34: 974-979

44 Tjernström F, Nyström A, Magnusson M. How to uncover the covert saccade during the head impulse test. Otol Neurotol 2012; 33: 1583-1585

45 Kattah JC, Talkad AV, Wang DZ et al. HINTS to diagnose stroke in the acute vestibular syndrome: three-step bedside oculomotor examination more sensitive than early MRI diffusion-weighted imaging. Stroke 2009; 40: 3504-3510

46 Cnyrim CD, Newman-Toker D, Karch C et al. Bedside differentiation of vestibular neuritis from central "vestibular pseudoneuritis". J Neurol Neurosurg Psychiatry 2008; 79: 458-460

47 Newman-Toker DE, Kattah JC, Alvernia JE, Wang DZ. Normal head impulse test differentiates acute cerebellar strokes from vestibular neuritis. Neurol 2008; 10: 2378-2385

48 Strupp M, Brandt T. Diagnosis and Treatment of Vertigo and Dizziness. Dtsch Arztebl Int 2008; 105: 173-180

49 Walther LE, Brusis T. From the expert's office: Current diagnosis and assessment of vestibular receptor function. Laryngorhinootol 2013; 92: 189-192

50 Vital D, Hegemann SC, Straumann D et al. A new dynamic visual acuity test to assess peripheral vestibular function. Arch Otolaryngol Head Neck Surg 2010; 136: 686-691

51 Leitlinie Schwindel-Diagnostik der Deutschen Gesellschaft für Neurologie. AWMF-Leitlinien Register 030/017, 4. überarbeitete Aufl. 2008

52 Walther LE. Dysequilibrium. Restorative management in disequilibrium. Laryngorhinootol 2005; 84 (Suppl 1): 70-91

53 Fife TD 1, Iverson DJ, Lempert T et al. Quality Standards Subcommittee, American Academy of Neurology. Practice parameter: therapies for benign paroxysmal positional vertigo (an evidence-based review): report of the Quality Standards Subcommittee of the American Academy of Neurology. Neurol 2008; 27: 2067-2074 
54 Gürkov R, Kantner C, Strupp M, Flatz W, Krause E, Ertl-Wagner B. Endolymphatic hydrops in patients with vestibular migraine and auditory symptoms. Eur Arch Otorhinolaryngol 2013 Oct 12 [Epub ahead of print]

55 Brandt T, Huppert T, Hüfner K et al. Long-term course and relapses of vestibular and balance disorders. Restor Neurol Neurosci 2010; 28: 69-82

56 Huppert D, Strupp M, Brandt T. Long-term course of Menière's disease revisited. Acta Otolaryngol 2010; 130: 644-651

57 Salt AN, Plontke SK. Endolymphatic hydrops: pathophysiology and experimental models. Otolaryngol Clin North Am 2010; 43: 971-983

58 Merchant SN, Adams JC, Nadol JB Jr. Pathophysiology of Meniere's syndrome: are symptoms caused by endolymphatic hydrops? Otol Neurotol 2005; 26: 74-81

59 Yamamoto $M$, Teranishi $M$, Naganawa $S$ et al. Relationship between the degree of endolymphatic hydrops and electrocochleography. Audiol Neurootol 2009; 15: 254-260

60 Klockhoff I, Lindblom U. Glycerol test in Ménière's disease. Acta Otolaryngol 1966; 27 (Suppl 224): 449ff

61 Nakashima T, Naganawa S, Sugiura M et al. Visualization of endolymphatic hydrops in patients with Meniere's disease. Laryngoscope 2007; 117: 415-420

62 Gürkov R, Flatz W, Louza J et al. In vivo visualized endolymphatic hydrops and inner ear functions in patients with electrocochleographically confirmed Ménière's disease. Otol Neurotol 2012; 33: 1040-1045

63 Naganawa S, Yamazaki M, Kawai H et al. MR Imaging of Ménière's Disease after Combined Intratympanic and Intravenous Injection of Gadolinium using HYDROPS2. Magn Reson Med Sci 2014; 28 [Epub ahead of print]

64 Schmid-Priscoveanu A, Böhmer A, Obzina H, Straumann $D$. Caloric and search-coil head-impulse testing in patients after vestibular neuritis. J Assoc Res Otolaryngol 2001; 2: 72-78

65 Palla A, Straumann D. Recovery of the high-acceleration vestibulo-ocular reflex after vestibular neuritis. J Assoc Res Otolaryngol 2004; 5: 427-435

66 Strupp M, Brandt T. Vestibular neuritis. Semin Neurol 2009; 29: 509-519

67 Walther LE, Hentschel H, Oehme A et al. Herpesviren bei akuter Innenohrschwerhörigkeit und „Vestibularisausfall“: klinische und serologische Befunde. Otorhinolaryngol Nova 2002; 12: 124-131

68 Halmagyi GM, Karlberg M, Curthoys IS, Todd MJ. Inferior vestibular neuritis. Ann N Y Acad Sci 2002; 956: 306-313

69 Walther LE, Repik I. Inferior vestibular neuritis: diagnosis using VEMP. HNO 2012; 60: 126-131

70 Park HJ, Migliaccio AA, Della Santina CC et al. Searchcoil head-thrust and caloric tests in Ménière's disease. Acta Otolaryngol 2005; 125: 852-857

$71 \mathrm{Kim} \mathrm{HA}$, Hong JH, Lee $\mathrm{H}$ et al. Otolith dysfunction in vestibular neuritis: recovery pattern and a predictor of symptom recovery. Neurol 2008; 70: 449-453

72 Park HK, Kim JS, Strupp M, Zee DS. Isolated floccular infarction: impaired vestibular responses to horizontal head impulse. J Neurol 2013; 260: 1576-1582

73 Wenzel A, Hülse R, Walther LE. Aktuelle Aspekte zum Dehiszenzsyndrom. Forum HNO 1/2013

74 Ward BK, Wenzel A, Ritzl EK et al. Near-dehiscence: clinical findings in patients with thin bone over the superior semicircular canal. Otol Neurotol 2013; 34: 1421-1428

75 Rosahl S, Samii M. Tumoren des Kleinhirnbrückenwinkels. In: Moskopp D, Wassmann H, Hrsg. Neurochirurgie. Stuttgart: Schattauer, 2004; 461-472

76 Walther LE, Blödow A. Ocular vestibular evoked myogenic potential to air conducted sound stimulation and video head impulse test in acute vestibular neuritis. Otol Neurotol 2013; 34: 1084-1089

77 Walther LE, Prosowsky K, Walther A, Gudziol H. Herpes zoster oticus: symptom constellation and serological diagnosis. Laryngo-Rhino-Otol 2004; 83: 355-362

\section{Über die Autoren}

$\nabla$

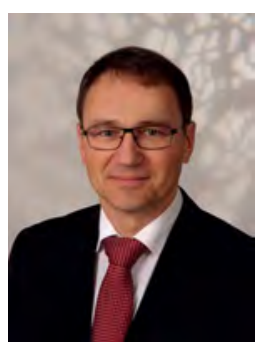

Stefan K. Plontke

Prof. Dr. med., Jahrgang 1968, studierte von 19901997 an der Humboldt-Universität Berlin (Charitè) sowie mit Stipendien an der University of Aberdeen (UK) und an der University of Pennsylvania (USA). Die Weiterbildung zum HNO-Facharzt erfolgte an der Universität Tübingen (2003 Facharzt), 2005 Ernennung zum Oberarzt, 2006 Habilitation und 2009 Ernennung zum außerplanmäßigen Professor. 2010 erfolgte der Ruf an die Martin-LutherUniversität Halle-Wittenberg, deren Universitäts-HNO-Klinik er als Direktor seit 2010 leitet. Prof. Plontke ist Vorstandsmitglied der ADANO der DGHNO, Mitglied des Board of Directors der Prosper Menière Society und weiterer Fachgesellschaften. Wissenschaftliche Schwerpunkte liegen im Bereich Hörforschung und klinische Studien.

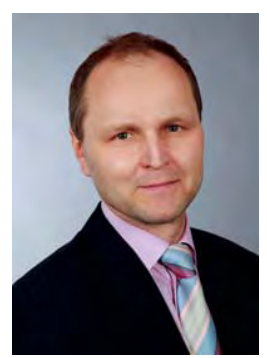

Leif Erik Walther

Prof. Dr. med., Jahrgang 1966, studierte von 19881994 an der Friedrich-Schiller-Universität Jena. Auslandsaufenthalt an der University of California (UCSF) in San Francisco 1991-1992. Facharzt 2000, 2003-2005 Oberarzt am Universitätsklinikum Aachen, Habilitation 2004. Seit 2006 in niedergelassener Tätigkeit in Sulzbach (Taunus), 2013 Hofmann- und Heermann-Preisträger der Deutschen Gesellschaft für HNO-Heilkunde, 2013 Ernennung zum außerplanmäßigen Professor der RuprechtKarls-Universität Heidelberg. Prof. Walther ist Mitglied ADANO der DGHNO sowie der American Academy of Otolaryngology, Head \& Neck Surgery. Wissenschaftliche Schwerpunkte liegen im Bereich der Erforschung von Otokonien und der klinischen Vestibularisdiagnostik.

\section{Korrespondenzadresse}

Prof. Dr. med. Stefan Plontke

Kopf- und Hals-Chirurgie

Universitätsklinik und Poliklinik für

Hals-Nasen-Ohren-Heilkunde

Martin-Luther-Universität Halle-

Wittenberg

Ernst-Grube-Straße 40

06120 Halle (Saale)

stefan.plontke@uk-halle.de 


\section{CME-Fragen Differenzialdiagnose „Schwindel“}

1 Welche Erkrankung ist durch episodische Schwindelattacken $<1$ min charakterisiert? Neuritis vestibularis

(Kanalolithiasis) des hinteren Bogengangs benigner paroxysmaler Lagerungsschwindel nichtkompensierte periphere Vestibulopathie Zoster oticus

Welche Aussage zur Definition des Morbus Menière ist falsch? Es werden mindestens 2 Schwindelanfälle $>20$ min gefordert.

B Der „Schwindel“ imponiert subjektiv meistens als Drehgefühl.

C Fluktuierender Tinnitus muss vorhanden sein.

D Fluktuierende Hörstörungen müssen vorhanden sein.

E Isolierte Hörstörungen im Tieftonbereich sind immer Zeichen eines Morbus Menière.

\section{Welche Aussage zur Definition von „Schwindel“ ist falsch?}

„Schwindel“ ist eine gestörte Wahrnehmung der Bewegung des eigenen Körpers.

B Bei „Schwindel“ ist die Wahrnehmung der Umgebung („äußerer Schwindel“) gestört.

C Bei „Schwindel“ besteht das Gefühl der Stand- und Gangunsicherheit.

D „Schwindel“ ist ein Gefühl, das nur im Zusammenhang mit einseitigen Hörstörungen auftritt.

E Die Wahrnehmung der räumlichen Orientierung ist im Rahmen von „Schwindel“ beeinträchtigt.

4 Welche Erkrankungen mit „Schwindel“ treten vorzugsweise häufig im Kindes- und Jugendalter auf? vestibuläre Migräne, orthostatische Dysregulation benigner paroxysmaler Lagerungsschwindel Cholesteatom

Zoster oticus

Morbus Menière und Neuritis vestibularis

\section{Bei welcher Erkrankung tritt keine Synkope auf?}

orthostatische Dysregulation

Neuritis vestibularis

Herzrhythmusstörungen

Myokardinfarkt

Hypoglykämie bei Diabetes mellitus
Welche Erkrankung kommt differenzialdiagnostisch in Frage? Ein Patient klagt über episodische Drehschwindelattacken, ein Tullio-Phänomen, Autophonie sowie Druck- und Völlegefühl im Ohrbereich.

A Morbus Menière

B multisensorischer Schwindel im höheren Lebensalter

C Dehiszenzsyndrom

D orthostatische Dysregulation

E Neuritis vestibularis

7 Welche anamnestischen Fragen sind bei „Schwindel“ und Stürzen im höheren Lebensalter nicht vordergründig von Bedeutung?

A Welche Medikamente werden eingenommen und in welcher Dosis?

B Kamen in der Vergangenheit Stürze oder „Beinahe“Stürze vor?

c Sind Hörstörungen und depressiven Erkrankungen vorhanden?

D Bestehen ein Gallensteinleiden und erhöhte Leberwerte?

E Ist das Sehvermögen beeinträchtigt?

Welches Symptom ist für eine Neuritis vestibularis untypisch?

akutes Drehgefühl

begleitende Fazialisparese, Schluckstörungen

vegetative Symptome

Stand- und Gangunsicherheit

reizloser Trommelfellbefund

9 Welche Aussage zu Augenbewegungsstörungen und Nystagmus trifft nicht zu?

A Bei peripheren Augenbewegungsstörungen ist meistens nur 1 Auge betroffen.

B Ein vertikaler Nystagmus hat meist eine zentrale Ursache.

C Ein Spontannystagmus ist immer peripher vestibulär bedingt.

D Die visuelle Fixation eines horizontal rotierenden Spontannystagmus spricht für eine periphere Vestibulopathie.

E Bei zentralen vestibulären Störungen kann ein Spontannystagmus vorhanden sein.

Welche Aspekte sind für den benignen paroxysmalen Lagerungsschwindel untypisch?

A Die Richtung des Nystagmus entspricht einer Augendrehachse, die senkrecht zur Ebene des erregten Bogengangs liegt.

B Ein pBPPV tritt meistens im höheren Lebensalter auf.

C Die Latenz des Nystagmus (VOR) beträgt ca. 5-15s.

D $\quad$ kurze Dauer ( $<1$ min, bei der Kupulolithiasis auch länger) und Ermüdbarkeit

E vorhandener peripherer Spontannystagmus, Dauerschwindel 


\section{CME-Fortbildung mit der Laryngo-Rhino-Otologie}

Zertifizierte Fortbildung Hinter der Abkürzung CME verbirgt sich „continuing medical education“, also kontinuierliche medizinische Fort- und Weiterbildung. Zur Dokumentation der kontinuierlichen Fortbildung der Ärzte wurde das Fortbildungszertifikat der Ärztekammern etabliert. Hauptzielgruppe für das Fortbildungszertifikat sind Ärzte mit abgeschlossener Facharztausbildung, die im 5-jährigen Turnus einen Fortbildungsnachweis erbringen müssen. Es ist jedoch auch für Ärzte in der Facharztweiterbildung gedacht.

Die Fortbildungseinheit In den einheitlichen Bewertungskriterien der Bundesärztekammer ist festgelegt: „Die Grundeinheit der Fortbildungsaktivitäten ist der Fortbildungspunkt. Dieser entspricht in der Regel einer abgeschlossenen Fortbildungsstunde (45 Minuten)“.

Für die erworbenen Fortbildungspunkte muss ein Nachweis erbracht werden. Hat man die erforderliche Anzahl von 250 Punkten gesammelt, kann man das Fortbildungszertifikat bei seiner Ärztekammer beantragen, welches man wiederum bei der KV (niedergelassene Ärzte) oder bei seinem Klinikträger (Klinikärzte) vorlegen muss.

Anerkennung der CME-Beiträge Die Fortbildung in der Laryngo-Rhino-Otologie wurde von der Nordrheinischen Akademie für Ärztliche Fort- und Weiterbildung für das Fortbildungszertifikat anerkannt, das heißt, die Vergabe der Punkte kann direkt durch die Thieme Verlagsgruppe erfolgen. Die Fortbildung in der Laryngo-Rhino-Otologie gehört zur Kategorie „strukturierte interaktive Fortbildung“. Entsprechend einer Absprache der Ärztekammern werden die von der Nordrheinischen Akademie für Ärztliche Fort- und Weiterbildung anerkannten Fortbildungs- veranstaltungen auch von den anderen zertifizierenden Ärztekammern anerkannt.

Datenschutz Ihre Daten werden ausschließlich für die Bearbeitung dieser Fortbildungseinheit verwendet. Es erfolgt keine Speicherung der Ergebnisse über die für die Bearbeitung der Fortbildungseinheit notwendige Zeit hinaus. Die Daten werden nach Versand der Testate anonymisiert. Namens- und Adressangaben dienen nur dem Versand der Testate. Die Angaben zur Person dienen nur statistischen Zwecken und werden von den Adressangaben getrennt und anonymisiert verarbeitet.

Teilnahme Jede Ärztin und jeder Arzt soll das Fortbildungszertifikat erlangen können. Deshalb ist die Teilnahme am CME-Programm der Laryngo-Rhino-Otologie nicht an ein Abonnement geknüpft! Die Teilnahme ist im Internet (http://cme.thieme.de) möglich.

Im Internet ist eine Registrierung erforderlich, wobei die Teilnahme an Fortbildungen abonnierter Zeitschriften ohne Zusatzkosten möglich ist.

Teilnahmebedingungen Für eine Fortbildungseinheit erhalten Sie 3 Fortbildungspunkte im Rahmen des Fortbildungszertifikates. Hierfür müssen 70\% der Fragen richtig beantwortet sein.

CME-Wertmarke für Nicht-Abonnenten Teilnehmer, die nicht Abonnenten der Laryngo-Rhino-Otologie sind, können für die Internet-Teilnahme dort direkt ein Guthaben einrichten, von dem pro Teilnahme ein Unkostenbeitrag abgebucht wird.

Teilnahme online möglich unter http://cme.thieme.de 